\title{
Leveraging of Rifampicin-Dosed Cynomolgus Monkeys to Identify Bile Acid 3-O-Sulfate Conjugates as Potential Novel Biomarkers for Organic Anion-Transporting Polypeptides ${ }^{\circledR}$
}

\author{
Rhishikesh Thakare, Hongying Gao, Rachel E. Kosa, Yi-An Bi, Manthena V. S. Varma, \\ Matthew A. Cerny, Raman Sharma, Max Kuhn, Bingshou Huang, Yiping Liu, Aijia Yu, \\ Gregory S. Walker, Mark Niosi, Larry Tremaine, Yazen Alnouti, and A. David Rodrigues
}

Pharmacokinetics, Dynamics \& Metabolism, Medicine Design (H.G., R.E.K., Y.B., M.V.S.V., M.A.C., R.S., G.S.W., M.N., L.T., A.D.R.), and Nonclinical Statistics, Pfizer Inc., Groton, Connecticut (M.K.); Department of Pharmaceutical Sciences, College of Pharmacy, University of Nebraska Medical Center, Omaha, Nebraska (R. T., Y. A.); and WuXi AppTec, Waigaoqiao Free Trade Zone, Shanghai, China (B. H., Y. L., A.Y.)

Received January 28, 2017; accepted April 5, 2017

\section{ABSTRACT}

In the search for novel bile acid (BA) biomarkers of liver organic anion-transporting polypeptides (OATPs), cynomolgus monkeys received oral rifampicin (RIF) at four dose levels $(1,3,10$, and $30 \mathrm{mg} / \mathrm{kg}$ ) that generated plasma-free $C_{\max }$ values $(0.06,0.66,2.57$, and $7.79 \mu \mathrm{M}$, respectively) spanning the reported in vitro $\mathrm{IC}_{50}$ values for OATP1B1 and OATP1B3 $(\leq 1.7 \mu \mathrm{M})$. As expected, the area under the plasma concentration-time curve (AUC) of an OATP probe drug (i.v. ${ }^{2} \mathrm{H}_{4}$-pitavastatin, $0.2 \mathrm{mg} / \mathrm{kg}$ ) was increased 1.2-, 2.4-, 3.8-, and 4.5-fold, respectively. Plasma of RIF-dosed cynomolgus monkeys was subjected to a liquid chromatography-tandem mass spectrometry method that supported the analysis of $\mathbf{3 0}$ different BAs. Monkey urine was profiled, and we also determined that the impact of RIF on BA renal clearance was minimal. Although sulfated BAs comprised only $1 \%$ of the plasma BA pool, a robust RIF dose response (maximal $\geq 50$-fold increase in plasma AUC) was observed for the sulfates of five BAs [glycodeoxycholate (GDCA-S), glycochenodeoxycholate (GCDCA-S), taurochenodeoxycholate, deoxycholate (DCA-S), and taurodeoxycholate (TDCA-S)]. In vitro, RIF ( $\leq 100 \mu \mathrm{M})$ did not inhibit cynomolgus monkey liver cytosol-catalyzed BA sulfation and cynomolgus monkey hepatocytemediated uptake of representative sulfated BAs (GDCA-S, GCDCA-S, DCA-S, and TDCA-S) was sodium-independent and inhibited ( $270 \%)$ by RIF (5 $\mu \mathrm{M})$; uptake of taurocholic acid was sensitive to sodium removal ( $74 \%$ decrease) and relatively refractory to RIF ( $\leq 21 \%$ inhibition). We concluded that sulfated BAs may serve as sensitive biomarkers of cynomolgus monkey OATPs and that exploration of their utility as circulating human OATP biomarkers is warranted.

\section{Introduction}

It is now accepted that organic anion-transporting polypeptides (OATPs) mediate the active uptake of numerous drugs into hepatocytes and hence govern their pharmacokinetic profile and liver (free)-toplasma (free) concentration ratio. OATPs can also serve as the loci of important drug-drug interactions (DDIs) leading to changes in systemic and local drug concentrations, possibly resulting in altered efficacy and toxicity profiles (Giacomini et al., 2010; Yoshida et al., 2012). Consequently, tools have been developed to facilitate OATP inhibition

https://doi.org/10.1124/dmd.117.075275.

S This article has supplemental material available at dmd.aspetjournals.org. screening in vitro, drive model-based DDI in vitro-in vivo extrapolations, and support OATP DDI risk assessment before human dosing (Poirier et al., 2007; Jamei et al., 2014; Vaidyanathan et al., 2016; Yoshikado et al., 2016). The latter is particularly important because OATP activity and expression are also known to be impacted by genotype and liver disease (Gong and Kim, 2013; Clarke et al., 2014).

More recently, it has been envisioned that OATP biomarkers will greatly facilitate clinical phenotyping and DDI studies while possibly deferring more formal studies using drug probes (Lai et al., 2016; Yee et al., 2016). For example, Lai et al. (2016) recently evaluated plasma bilirubin, bilirubin glucuronide, and coproporphyrin isomers (I and III) as OATP biomarkers in human subjects after a single dose of rifampicin (RIF). These authors noted a 4.0- and 3.3-fold increase in the

ABBREVIATIONS: AUC, area under the plasma concentration-time curve; $\mathrm{AUCR}_{\text {plasma }}, \mathrm{AUC}$ ratio determined by dividing the $\mathrm{AUC} \mathrm{C}_{0-24, \text { plasma }}$ after RIF treatment by the $\mathrm{AUC}_{0-24, \text { plasma }}$ after vehicle alone; BA, bile acid; CA, cholic acid; CA-S, cholic acid 3-O-sulfate; CDCA, chenodeoxycholic acid; CDCA-S, chenodeoxycholic acid 3-O-sulfate; $\mathrm{CL}_{\text {renal }}$ ratio, renal clearance; DCA-S, deoxycholic acid 3-O-sulfate; DDI, drug-drug interaction; DHEA, dehydroepiandrosterone; FDR, false discovery rate; GCA, glycocholic acid; GCA-S, glycocholic acid 3-O-sulfate; GCDCA, glycochenodeoxycholic acid; GCDCA-S, glycochenodeoxycholic acid 3-O-sulfate; GLCA, glycolithocholic acid; GLCA-S, glycolithocholic acid 3-O-sulfate; GUDCA, glycoursodeoxycholic acid; GUDCA-S, glycoursodeoxycholic acid 3-O-sulfate; LCA, lithocholic acid; LCA-S, lithocholic acid 3-O-sulfate; LC-MS/MS, liquid chromatography-tandem mass spectrometry; NTCP, sodium-taurocholate cotransporting polypeptide; OAT3, organic anion transporter 3; OATP, organic anion-transporting polypeptide; PAPS, 3'-phosphoadenosine-5' -phosphosulfate; QC, quality control; RIF, rifampicin; RIFsv, rifamycin SV; SLC, solute carrier; SULT2A1, sulfotransferase 2A1; TCA, taurocholic acid; TCA-S, taurocholic acid 3-Osulfate; TCDCA, taurochenodeoxycholic acid; TCDCA-S, taurochenodeoxycholic acid 3-O-sulfate; TDCA, taurodeoxycholic acid; TDCA-S, taurodeoxycholic acid 3-O-sulfate; TLCA, taurolithocholic acid; TLCA-S, taurolithocholic acid 3-O-sulfate; TUDCA, tauroursodeoxycholic acid; TUDCA-S, tauroursodeoxycholic acid 3-O-sulfate; UDCA, ursodeoxycholic acid; UDCA-S, ursodeoxycholic acid 3-O-sulfate. 
coproporphyrin I and III area under the plasma concentration-time curve (AUC), respectively, consistent with in vitro data (Bednarczyk and Boiselle, 2016; Shen et al., 2016). Likewise, Yee et al. (2016) identified the 3-O-sulfate conjugates of glycochenodeoxycholic acid (GCDCA-S), glycodeoxycholic acid (GDCA-S), and taurolithocholic acid (TLCA-S) as candidate OATP biomarkers after dosing of SLCO1B1 genotyped subjects with cyclosporine.

Because of the high sequence identity with human OATPs, the cynomolgus monkey has been used increasingly as a model to study OATP inhibition in vivo (Shen et al., 2013; Takahashi et al., 2013; Shen et al., 2015). The utility of the cynomolgus monkey has extended also to the search for OATP biomarkers, which has involved the administration of a single RIF dose and reporting its impact on plasma bilirubin, bilirubin glucuronide, coproporphyrins (I and III), nonsulfated bile acids (BAs), and dehydroepiandrosterone (DHEA) 3-O-sulfate (Chu et al., 2015; Watanabe et al., 2015; Shen et al., 2016).

As described, an attempt was made to extend the work of Chu et al. (2015) by profiling 30 different BAs in cynomolgus monkey plasma after single oral doses of $\operatorname{RIF}(1,3,10$, and $30 \mathrm{mg} / \mathrm{kg})$. It was possible to prepare synthetic standards of numerous BA 3-O-sulfates and apply a liquid chromatography-tandem mass spectrometry (LC-MS/MS) method that has been used successfully to profile nonsulfated and sulfated BAs in human serum and urine (Bathena et al., 2013). It should be noted that at the time of the study, BA sulfation in the cynomolgus monkey was not well characterized, although the putative hydroxysteroid sulfotransferase (SULT2A1) that catalyses BA sulfation in humans was known to be expressed in monkey liver (Nishimura et al., 2008; Alnouti, 2009; Nishimura et al., 2009). It was also known that DHEA 3-O-sulfate was detectable in cynomolgus monkeys after DHEA administration (Leblanc et al., 2003).

In the present study, animals also received a single i.v. dose of ${ }^{2} \mathrm{H}_{4}$ pitavastatin to ensure that OATP was inhibited by RIF in a dosedependent manner (Takahashi et al., 2013). In addition, the plasma concentrations of RIF were determined at each of the four dose levels. Sulfated BAs are known to be cleared renally in humans (Alnouti, 2009; Bathena et al., 2013; Tsuruya et al., 2016); therefore, BA profiling was extended to include urine of control and RIF-dosed cynomolgus monkeys. The different BAs were then assessed in terms of their utility as OATP biomarkers: 1) detectability in control animals; 2) magnitude of the RIF dose response; and 3) detectability in human serum. Based on these criteria, seven sulfated BAs [GCDCA-S, GDCA-S, glycolithocholate 3-O-sulfate (GLCA-S), TLCA-S, taurochenodeoxycholate 3-O-sulfate (TCDCA-S), taurodeoxycholate 3-O-sulfate (TDCA-S), and deoxycholate 3-O-sulfate (DCA-S)] were identified as potential OATP biomarkers. For GCDCA-S, GDCA-S, and TLCA-S, the results are consistent with human plasma metabolomic data from a recent OATP1B1 (SLCO1B1) genome-wide association study and earlier reports describing TLCA-S as an OATP substrate in vitro (Meng et al., 2002; Sasaki et al., 2002; Yee et al., 2016). The present study also included an in vitro assessment of BA sulfation by cynomolgus monkey liver cytosol, as well as uptake studies with GDCA-S, GCDCA-S, DCA-S, and TDCA-S (cynomolgus monkey plated primary hepatocytes) to phenotype both in terms of OATP- and sodium-taurocholate co-transporting polypeptide (NTCP)-mediated active uptake.

\section{Materials and Methods}

\section{Chemicals and Reagents}

3'-Phosphoadenosine-5'-phosphosulfate (PAPS), simvastatin, rifamycin SV (RIFsv), and RIF were purchased from Sigma-Aldrich (St. Louis, MO). Deuterium-labeled RIF $\left({ }^{2} \mathrm{H}_{8}\right.$-RIF) was obtained from ALSACHIM (Illkirch, Graffenstaden, France). Pitavastatin was purchased from Sequoia Research
Products Ltd. (Oxford, UK). Deuterium-labeled pitavastatin $\left({ }^{2} \mathrm{H}_{4}\right.$-pitavastatin) was purchased from Clearsynth Canada Inc (Mississauga, ON, Canada). InVitroGro-HT and CP hepatocyte media were purchased from Celsis IVT (Baltmore, MD). Collagen I-coated 24-well plates were obtained from BD Biosciences (Franklin Lakes, NJ). Cryopreserved cynomolgus monkey hepatocytes were purchased from In vitro ADMET Laboratories, LLC (Columbia, MD). Bicinchoninic acid protein assay kit was purchased from Pierce (Rockford, IL). Methanol, acetonitrile, water, and ammonium hydroxide were obtained from Fisher Scientific (Fair Lawn, NJ)

Cholic acid (CA), glycocholic acid (GCA), taurocholic acid (TCA), deuteriumlabeled taurocholic acid $\left({ }^{2} \mathrm{H}_{4}\right.$-TCA), chenodeoxycholic acid (CDCA), deuterium-labeled chenodeoxycholic acid $\left({ }^{2} \mathrm{H}_{4}\right.$-CDCA), taurochenodeoxycholic acid (TCDCA), deoxycholic acid (DCA), glycochenodeoxycholic acid (GCDCA), glycodeoxycholic acid (GDCA), taurodeoxycholic acid (TDCA), lithocholic acid (LCA), glycolithocholic acid (GLCA), taurolithocholic acid (TLCA), ursodeoxycholic acid (UDCA), tauroursodeoxycholic acid (TUDCA), glycoursodeoxycholic acid (GUDCA), and deuterium-labeled glycodeoxycholic acid 3-O-sulfate $\left({ }^{2} \mathrm{H}_{4}\right.$-GDCA-S) were purchased from IsoSciences (King of Prussia, PA). Lithocholic acid 3-O-sulfate (LCA-S), glycolithocholic acid 3-O-sulfate (GLCA-S), chenodeoxycholic acid 3-O-sulfate (CDCA-S), glycochenodeoxycholic acid 3-O-sulfate (GCDCA-S), and deuterium-labeled glycochenodeoxycholic acid 3-O-sulfate $\left({ }^{2} \mathrm{H}_{5}\right.$-GCDCA-S) were purchased from Toronto Research Chemicals (Toronto, ON, Canada). Ursodeoxycholic acid 3-O-sulfate (UDCA-S) was obtained from ALSACHIM (Illkirch, Graffenstaden, France). Deuterium labeled glycochenodeoxycholic acid $\left({ }^{2} \mathrm{H}_{4}\right.$-GCDCA) was purchased from $\mathrm{C} / \mathrm{D} / \mathrm{N}$ isotopes, Inc. (Pointe-Claire, Quebec, Canada). Taurolithocholic acid 3-O-sulfate (TLCA-S) was purchased from SigmaAldrich (St. Louis, MO)

Refer to the Supplemental Material for the chemical synthesis of taurochenodeoxycholic acid 3-O-sulfate (TCDCA-S), deoxycholic acid 3-Osulfate (DCA-S), glycodeoxycholic acid 3-O-sulfate (GDCA-S), taurodeoxycholic acid 3-O-sulfate (TDCA-S), cholic acid 3-O-sulfate (CA-S), glycocholic acid 3-O-sulfate (GCA-S), taurocholic acid 3-O-sulfate (TCA-S), and biosynthesis of glycoursodeoxycholic acid 3-O-sulfate (GUDCA-S) and tauroursodeocycholic acid 3-O-sulfate (TUDCA-S). Biosynthesis of ${ }^{2} \mathrm{H}_{4}$-TDCA-S and ${ }^{2} \mathrm{H}_{4}$-DCA-S is described also. An alternative method for preparing some of the BA sulfates described herein has been described by Donazzolo et al. (2017). Refer to the Supplemental Material for all BA common names, chemical names, structures, and CAS numbers.

\section{Animal Handling, Dosing, Plasma Draws, and Urine Collection}

All experiments involving animals were conducted at the Pfizer Groton (Connecticut) facilities (Association for Assessment \& Accreditation of Laboratory Animal Care-Accredited) and were reviewed and approved by the Pfizer Institutional Animal Care and Use Committee. Male cynomolgus macaque Mauritian monkeys (approximately 6-8.5 years of age) were used for these studies. A crossover study design was used, in which the same four animals were dosed over a series of five studies, after a minimum 1-week washout period between each study. One exception was the $3 \mathrm{mg} / \mathrm{kg} \mathrm{RIF}$ dose group, in which one of four monkeys was dosed only in that single study.

Animals were provided a normal food schedule the day before the study (meals at 8:00 AM and 11:00 AM, with one treat daily) and were allowed free access to water. On the day of the study, monkeys were fed at approximately 1 and 3 hours postdose and allowed water ad libitum. RIF was administered via oral gavage at 0 (blank vehicle), 1, 3, 10, and $30 \mathrm{mg} / \mathrm{kg}$. RIF was given at a dose volume of $2 \mathrm{ml} / \mathrm{kg}$ in a $0.5 \%(\mathrm{w} / \mathrm{v})$ methylcellulose (in water) suspension. Approximately 1 hour and 15 minutes after the oral RIF administration, ${ }^{2} \mathrm{H}_{4}$-pitavastatin was administered via an i.v. bolus (cephalic vein) at dose of $0.2 \mathrm{mg} / \mathrm{kg}$ in a dosing volume of $0.2 \mathrm{ml} / \mathrm{kg}, 2 \%$ DMSO (v/v), and $98 \%$ of TRIS-buffered saline $(\mathrm{pH} \sim 7.7)$. All i.v. formulations were sterile filtered before administration. Serial blood samples were collected via the femoral vein before collecting in $\mathrm{K}_{2}$ EDTA tubes and then at $0.083,0.25,0.5,0.75,1,2,3,5,6$, and 24 hours after i.v. dosing. Blood samples were stored on wet ice before being centrifuged to obtain plasma (3000 RPM, 10 minutes at $4{ }^{\circ} \mathrm{C}$; Jouan BR4i refrigerated centrifuge). Urine was also collected (metabolism cages) on wet ice, predose and at intervals of 0-6 hours and 6-24 hours postdose. Owing to instability and possible interconversion of lactone to pitavastatin, each plasma and urine sample was equally divided into two 
aliquots before being stored frozen. The first was untreated matrix, and the second was added to an equal volume of $0.1 \mathrm{M}$ sodium acetate buffer $(\mathrm{pH} 4)$ to stabilize pitavastatin. All urine and plasma samples, treated and untreated, were kept cold during collection, after which they were stored frozen at $-20^{\circ} \mathrm{C}$. It is known that BAs undergo enterohepatic recirculation, but no attempt was made to collect portal vein blood from the different cynomolgus monkeys. After LC-MS/MS analysis of femoral vein-derived plasma, it was apparent that the concentration (total plasma) of the various BA sulfates was low $(\leq 30 \mathrm{nM})$. For some of the BA sulfates (TCDCA-S, GCDCA-S,TDCA-S, and GDCA-S), unbound fraction in cynomolgus monkey plasma was determined $(\sim 0.016)$; maximal free plasma concentration was $\sim 0.5 \mathrm{nM}$. It is assumed that even if free BA sulfate concentrations were 100-fold higher in the portal vein, such concentrations would still likely be below the apparent $K_{\mathrm{m}}$ for cynomolgus monkey OATPs. As described in the Results, RIF dosing brought about robust ( $\geq 10$-fold) dose-dependent increase in the area under the plasma concentration-time curve (AUC) for a number of BA sulfates (LCA-S, GLCA-S, TLCA-S, GCDCA-S, TCDCA-S, DCA-S, GDCA-S, TDCA-S). Such a result is consistent with low substrate concentration-to-OATP $K_{\mathrm{m}}$ ratios in vivo.

\section{LC-MS/MS) Analysis}

Analysis of BAs and their 3-O-Sulfate Conjugates. Plasma and urine concentrations of BAs and their 3-O-sulfate conjugates were measured in untreated matrices by LC-MS/MS, as described previously, with some modifications (Bathena et al., 2013). Briefly, a Waters ACQUITY ultraperformance liquid chromatography (UPLC) system (Waters, Milford, MA) was coupled to an 5500 Q TRAP quadrupole linear ion trap hybrid mass spectrometer (MS) with an electrospray ionization (ESI) source (Applied Biosystems, MDS Sciex, Foster City, CA). Chromatographic separations were performed using an ACQUITY UPLC BEH C18 column $(1.7 \mu \mathrm{m}, 150 \times 2.1 \mathrm{~mm})$ maintained at $25^{\circ} \mathrm{C}$ and equipped with an in-line precolumn filter. The mobile phase consisted of $7.5 \mathrm{mM}$ ammonium bicarbonate, adjusted to $\mathrm{pH} 9.0$ using ammonium hydroxide (mobile phase A) and 30\% acetonitrile in methanol (mobile phase B), at a total flow rate of $0.2 \mathrm{ml} / \mathrm{min}$. The gradient profile was held at $52.5 \%$ mobile phase B for 12.75 minutes, increased linearly to $68 \%$ in 0.25 minute, held at $68 \%$ for 8.75 minutes, increased linearly to $90 \%$ in 0.25 minute, held at $90 \%$ for 1 minute, and finally brought back to $52.5 \%$ in 0.25 minute followed by 4.75 minute re-equilibration (total run time of 28 minutes per sample). Ten microliters of sample was injected for analysis. Quantitative data were acquired in multiple reaction monitoring (MRM)-negative ESI mode. MRM transitions and MS parameters for the different BAs and their respective 3-O-sulfate conjugates are shown in Supplemental Table 1.

For preparation of calibration curves, blank plasma and urine were obtained by charcoal stripping as described previously (Bathena et al., 2013). Fourteenpoint calibration curves were prepared in stripped matrices by spiking $10 \mu \mathrm{l}$ of appropriate standard solution at final concentrations ranging from 0.5 to $2500 \mathrm{ng} / \mathrm{ml}$. For extraction of plasma samples, $1 \mathrm{ml}$ of ice-cold alkaline ACN (5\% $\mathrm{NH}_{4} \mathrm{OH}$ ) containing ${ }^{2} \mathrm{H}_{4}$-GCDCA and ${ }^{2} \mathrm{H}_{4}$-CDCA as internal standards was added to $100 \mu \mathrm{l}$ of samples. Samples were then vortex-mixed and centrifuged at
$16,000 \mathrm{~g}$ for 10 minutes, and the supernatants were aspirated, evaporated, and reconstituted in $100 \mu \mathrm{l}$ of $50 \% \mathrm{MeOH}$ solution. Urine samples were extracted similarly to plasma samples except Tween 20 was added (final concentration of $0.2 \% \mathrm{v} / \mathrm{v})$ to reduce nonspecific binding.

Analysis of ${ }^{2} \mathbf{H}_{\mathbf{4}}$-Pitavastatin and RIF. The plasma concentrations of RIF and ${ }^{2} \mathrm{H}_{4}$-pitavastatin were measured in plasma samples treated with $0.1 \mathrm{M}$ sodium acetate buffer ( $\mathrm{pH} 4)$ using the LC-MS/MS system listed herein. All standards and quality controls (QCs) were made in blank monkey plasma mixed with an equal volume of $0.1 \mathrm{M}$ sodium acetate buffer ( $\mathrm{pH} 4)$. Standard and QC mixtures of the analytes were made to encompass a range of concentrations $\left(0.1-500 \mathrm{ng} / \mathrm{ml},{ }^{2} \mathrm{H}_{4}\right.$ pitavastatin; $1-5000 \mathrm{ng} / \mathrm{ml}$, RIF). Samples were diluted to be measured in the linear range of the instrument responses, with high specificity of MRM (no interference in the blank matrixes) and a wide dynamic range for each analyte; the dilution integrity was confirmed by independent analysis of the drugs in the samples in separate assays. Aliquots of $50 \mu \mathrm{l}$ of standards, QCs, and plasma samples were prepared by protein precipitation using $200 \mu \mathrm{l}$ of acetonitrile containing an internal standard mixture of simvastatin and ${ }^{2} \mathrm{H}_{8}$ - RIF $(100 \mathrm{ng} / \mathrm{ml})$. The plates were vortexed for 2 minutes and centrifuged at $3000 \mathrm{rpm}$ for 10 minutes, and 100- $\mu 1$ supernatants of the mixture were transferred for LC-MS/MS analysis. Chromatographic separation was accomplished on a Waters Acquity UPLC HSS T3 C18 column $(1.8 \mu \mathrm{m}, 2.1 \times 50 \mathrm{~mm})$ maintained at $40^{\circ} \mathrm{C}$. The mobile phase consisted of two solvents, solvent $\mathrm{A}$ ( $0.1 \%$ formic acid in water) and solvent B $(0.1 \%$ formic acid in acetonitrile). The total run time for each injection was 3 minutes. The flow rate was $0.6 \mathrm{ml} / \mathrm{min}$. The gradient was maintained at $5 \%$ B for 0.3 minutes, followed by a linear increase to $95 \% \mathrm{~B}$ in 1.8 minutes, and kept at $95 \% \mathrm{~B}$ for 0.3 minute and then a linear decrease to $5 \%$ in 0.3 minutes. The column was equilibrated at $5 \% \mathrm{~B}$ for 0.3 minute. A Valco VICI valve (Valco Instruments Co., Houston, TX) was used to divert the first 0.3 minute and the last 0.5 minute of UPLC effluent to waste. The injection volume was $2 \mu \mathrm{l}$. The analytes were monitored using MRM with settings listed in Supplemental Table 2 .

\section{Determination of Uptake Clearance in the Presence of Cynomolgus Monkey Plated Primary Hepatocytes}

Thawing and seeding procedure for cynomolgus monkey hepatocytes was the same as that described previously for human hepatocytes (Bi et al., 2006). In brief, cryopreserved cynomolgus monkey primary hepatocytes (male animal; Lot no. 10106012; In vitro ADMET Laboratories, LLC. Columbia, MD) were thawed and seeded into 24-well collagen-coated plates using In Vitro-HT and In Vitro-CP hepatocyte media at a density of $0.35 \times 10^{6}$ cells/well $(0.5 \mathrm{ml} /$ well $)$. After culturing for 6 hours, the uptake study was conducted. To assess the rate of uptake and passive diffusion, the cells were princubated with and without (DMSO only) RIFsv $(1 \mathrm{mM})$ or RIF $(5 \mu \mathrm{M})$ at $37^{\circ} \mathrm{C}$ for 10 minutes. The uptake was initiated by the addition of $0.5 \mathrm{ml}$ containing ${ }^{2} \mathrm{H}_{4}$-TCDCA-S $(0.1 \mu \mathrm{M}),{ }^{2} \mathrm{H}_{4}$-TDCA-S $(0.1 \mu \mathrm{M}),{ }^{2} \mathrm{H}_{4}$-DCA-S $(0.1 \mu \mathrm{M}),{ }^{2} \mathrm{H}_{5}$-GCDCA-S $(0.1$ and $0.5 \mu \mathrm{M}),{ }^{2} \mathrm{H}_{4}$-GDCA-S $(0.1$ and $0.5 \mu \mathrm{M}),{ }^{2} \mathrm{H}_{4}$-TCA $(0.1$ and $0.5 \mu \mathrm{M})$, and nonlabeled pitavastatin $(0.1 \mu \mathrm{M})$. To determine the effect of sodium on substrate uptake, the cells were preincubated in Krebs-Henseleit buffer without sodium $\left(\mathrm{NaCl}\right.$ and $\mathrm{NaHCO}_{3}$

TABLE 1

Pharmacokinetic parameters of RIF after oral administration to male cynomolgus monkeys

\begin{tabular}{lcccc}
\hline \multirow{2}{*}{ Parameter } & \multicolumn{4}{c}{ RIF Dose } \\
\cline { 2 - 5 } & $1 \mathrm{mg} / \mathrm{kg}$ & $3 \mathrm{mg} / \mathrm{kg}$ & $10 \mathrm{mg} / \mathrm{kg}$ & $30 \mathrm{mg} / \mathrm{kg}$ \\
\hline $\mathrm{AUC}_{0-24, \text { plasama }}(\mathrm{ng} . \mathrm{h} / \mathrm{ml})$ & $985 \pm 396^{a}$ & $11300 \pm 3260$ & $70,100 \pm 10,800$ & $240,000 \pm 73,900$ \\
& $(1)^{b}$ & $(11)$ & $(71)$ & $(243)$ \\
Plasma total $C_{\max }(\mathrm{ng} / \mathrm{ml})$ & $176 \pm 99$ & $2050 \pm 245$ & $7980 \pm 2480$ & $24,200 \pm 14,100$ \\
& $(1)$ & $(12)$ & $(45)$ & $(137)$ \\
Plasma total $C_{\max }(\mu \mathrm{M})$ & $0.2 \pm 0.1$ & $2.5 \pm 0.3$ & $9.7 \pm 3.0$ & $29 \pm 17$ \\
Plasma free $C_{\max }(\mu \mathrm{M})^{c}$ & $0.06 \pm 0.03$ & $0.66 \pm 0.08$ & $2.57 \pm 0.80$ & $7.79 \pm 4.54$ \\
$T_{\max }(\mathrm{h})$ & $2.70 \pm 0.86$ & $1.80 \pm 0.36$ & $1.70 \pm 0.43$ & $2.70 \pm 0.58$ \\
$t_{1 / 2}(\mathrm{~h})$ & $5.18 \pm 1.52$ & $2.84 \pm 0.20$ & $4.56 \pm 2.18$ & $11.50 \pm 6.26$ \\
\hline
\end{tabular}

${ }^{a}$ Data are presented as mean \pm S.D. $(n=4$ animals $)$.

${ }^{b}$ Value in parentheses represents a ratio (vs. RIF at $1 \mathrm{mg} / \mathrm{kg}$ ).

${ }^{c}$ Plasma total $C_{\max }$ corrected for RIF plasma-free fraction (0.265). RIF plasma protein binding was determined using equilibrium dialysis with ascorbic acid as a stabilizer (see Supplemental Material). 
TABLE 2

Pharmacokinetic parameters of ${ }^{2} \mathrm{H}_{4}$-pitavastatin in male cynomolgus monkeys after i.v. administration $(0.2 \mathrm{mg} / \mathrm{kg})$ with increasing doses of RIF

\begin{tabular}{|c|c|c|c|c|c|}
\hline \multirow{2}{*}{ Parameter } & \multicolumn{5}{|c|}{ Oral RIF Dose $^{a}$} \\
\hline & Vehicle & $1 \mathrm{mg} / \mathrm{kg}$ & $3 \mathrm{mg} / \mathrm{kg}$ & $10 \mathrm{mg} / \mathrm{kg}$ & $30 \mathrm{mg} / \mathrm{kg}$ \\
\hline $\operatorname{AUC}_{0-24, \text { plasma }}(\mathrm{ng} \cdot \mathrm{h} / \mathrm{ml})$ & $192 \pm 49$ & $223 \pm 41$ & $459 \pm 141^{*}$ & $722 \pm 190^{*}$ & $862 \pm 319^{*}$ \\
\hline AUCR plasma $^{b}$ & 1.0 & 1.2 & $2.4 *$ & $3.8^{*}$ & $4.5^{*}$ \\
\hline $\mathrm{C}_{0}(\mathrm{ng} / \mathrm{ml})$ & $1200 \pm 628$ & $925 \pm 278$ & $1840 \pm 579$ & $1660 \pm 355$ & $1570 \pm 577$ \\
\hline$t_{1 / 2}(\mathrm{~h})$ & $5.95 \pm 0.85$ & $7.21 \pm 1.74$ & $2.95 \pm 1.86^{*}$ & $3.77 \pm 0.68$ & $4.55 \pm 0.59$ \\
\hline CL (ml/min per kilogram) & $18 \pm 4.6$ & $14.2 \pm 2.2$ & $7.6 \pm 2.0^{*}$ & $4.8 \pm 1.36^{*}$ & $4.3 \pm 1.61 *$ \\
\hline$V_{\mathrm{dSS}}($ liter $/ \mathrm{kg})$ & $1.79 \pm 0.72$ & $1.97 \pm 0.50$ & $0.47 \pm 0.2 *$ & $0.52 \pm 0.164 *$ & $0.55 \pm 0.20 *$ \\
\hline$\%$ Dose in urine ${ }^{c}$ & $1.8 \pm 2.0$ & $1.2 \pm 0.6$ & $1.2 \pm 1.3$ & $4.0 \pm 2.0$ & $2.9 \pm 0.4$ \\
\hline
\end{tabular}

${ }^{a}$ Data are presented as mean \pm S.D. $(n=4$ animals $)$

${ }^{b}$ Mean $\mathrm{AUCR}_{\text {plasma }}$ (RIF versus vehicle control).

${ }^{c} \%$ Total dose (as ${ }^{2} \mathrm{H}_{4}$-pitavastatin) recovered in urine over 24 hours.

*Statistically significant difference versus vehicle control arm; $P<0.05$ (one-way ANOVA, Dunnett multiple comparison method).

replaced with choline chloride and choline bicarbonate, respectively) at $37^{\circ} \mathrm{C}$ for 10 minutes (Ho et al., 2004). In all cases, incubations were terminated at $0.5,1,2$, and 5 minutes by washing the cells three times with ice-cold Hanks' balanced salt solution buffer. The cells were then lysed with $100 \%$ methanol containing internal standard (diclofenac), centrifuged, and dried down under nitrogen and reconstituted in 50:50 methanol-to-water ratio. Chromatography was performed on a Waters Acquity UPLC System (Milford, MA). The autosampler and column were kept at $10^{\circ} \mathrm{C}$ and $50^{\circ} \mathrm{C}$, respectively. Separation was achieved with a Waters $\mathrm{BEH}$ C18 column $(2.1 \times 50 \mathrm{~mm}, 1.7 \mu \mathrm{m})$ and a gradient of $7.5 \mathrm{mM}$ ammonium bicarbonate (mobile phase A) and 70:30 methanol/acetonitrile (mobile phase B) at a flow rate of $0.2 \mathrm{ml} / \mathrm{min}$. An initial mobile-phase composition of $50 \% \mathrm{~B}$ was held for 2 minutes, then ramped to $95 \%$ in 1.5 minutes, held at $95 \%$ for 1 minute, and returned to initial $50 \% \mathrm{~B}$ for 0.5 minute re-equilibration. The total analysis time for each sample was 5 minutes. Data were collected on an AB Sciex API5500
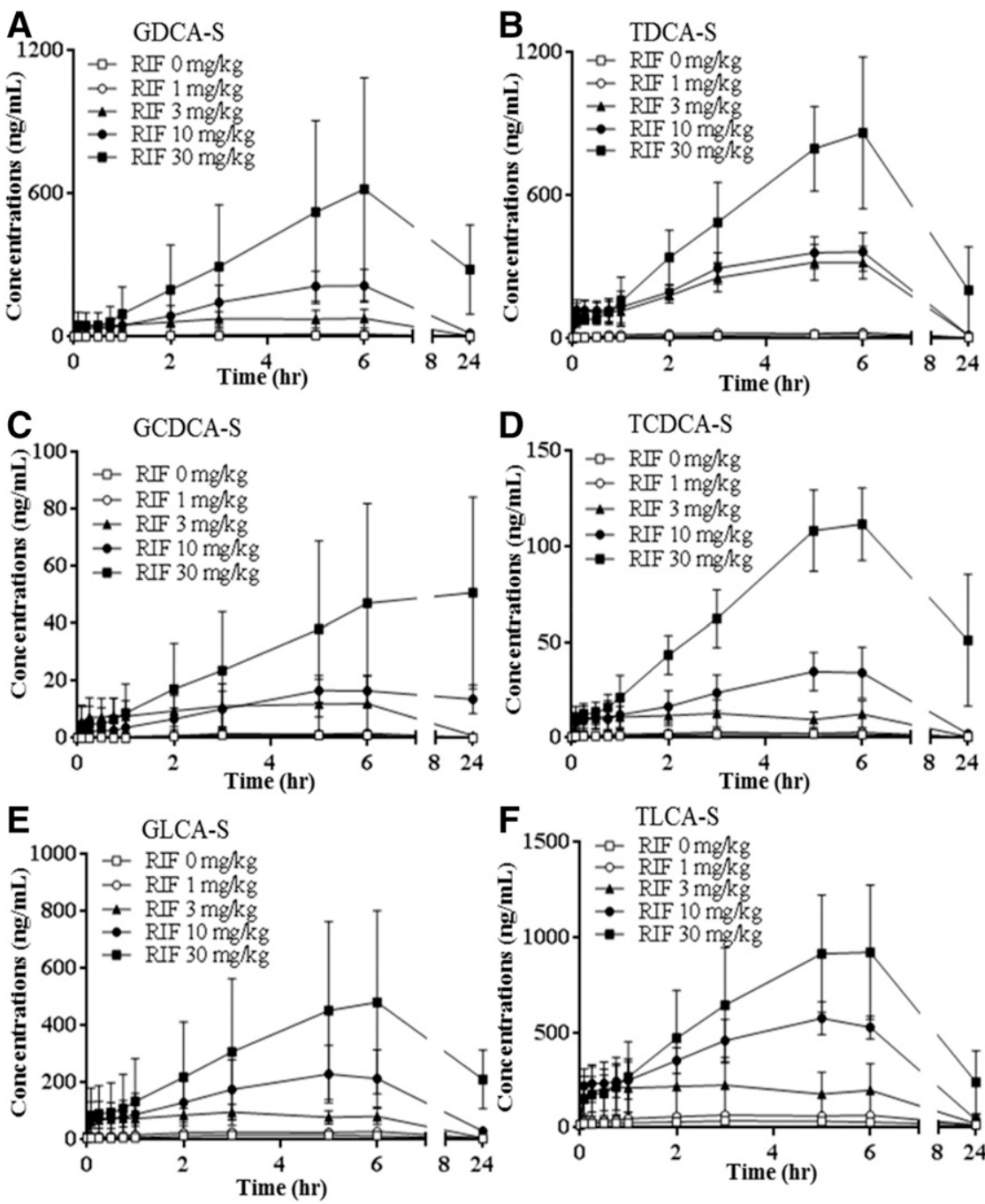

Fig. 1. Plasma concentration-time profile of (A) GDCA-S, (B) TDCA-S, (C) GCDCA-S, (D) TCDCA-S, (E) GLCA-S, and (F) TLCA-S in male cynomolgus monkeys after oral administration of vehicle alone (open squares) and RIF at $1 \mathrm{mg} / \mathrm{kg}$ (open circles), $3 \mathrm{mg} / \mathrm{kg}$ (closed triangles), $10 \mathrm{mg} / \mathrm{kg}$ (closed circles), and $30 \mathrm{mg} / \mathrm{kg}$ (closed squares). Data are presented as mean \pm S.D. $(n=4$ animals $)$. 
(QTRAP) mass spectrometer (Foster City, CA) using negative Turbo IonSpray ESI and MRM mode with the according to transitions: 290.6/96.8 ( ${ }^{2} \mathrm{H}_{4}$-TDCA-S $)$, 475.2/96.8 ( ${ }^{2} \mathrm{H}_{4}$-DCA-S $), 533.3 / 453.4\left({ }^{2} \mathrm{H}_{5}\right.$-GCDCA-S $), 532.3 / 452.3\left({ }^{2} \mathrm{H}_{4}\right.$-GDCA-S), and 514.2/79.8 $\left({ }^{2} \mathrm{H}_{4}\right.$-TCA). Data acquisition and processing were carried out using Analyst software version 1.6.2. (Applied Biosystems/MDS Sciex, Canada). Analysis of pitavastatin was performed as described here. Stability of the various sulfated BA substrates was confirmed after their addition to assay buffer and incubation for 5 minutes at $37^{\circ} \mathrm{C}$.

\section{BA Sulfation Catalyzed by PAPS-Fortified Cynomolgus Liver Cytosol}

Refer to the Supplemental Material for details related to incubation conditions and LC-MS/MS analysis.

\section{Pharmacokinetics Analysis}

BAs. For each BA, the plasma AUC from 0 to 24 hours $\left(\mathrm{AUC}_{0-24, \text { plasma }}\right)$ was derived from the concentration-time profile for each individual animal (trapezoidal rule, Microsoft Office Excel). Renal clearance $\left(\mathrm{CL}_{\text {renal }}\right)$ was calculated by dividing the amount excreted in urine from 0 to 24 hours $\left(\mathrm{A}_{\mathrm{e} 0-24 \text {,urine }}\right)$ by the $\mathrm{AUC}_{0-24, \text { plasma. }} \mathrm{AUC}_{0-24 \text {,plasma }}$ ratios ( $\mathrm{AUCR}_{\text {plasma }}$ ) were determined by dividing the $\mathrm{AUC}_{0-24, \text { plasma }}$ after RIF treatment by the vehicle alone $\mathrm{AUC}_{0-24, \text { plasma. }}$ The $\mathrm{CL}_{\text {renal }}$ ratio was determined by dividing the $\mathrm{CL}_{\text {renal }}$ after $\mathrm{RIF}$ treatment by the vehicle alone $\mathrm{CL}_{\text {renal }}$.

RIF and Pitavastatin. The noncompartmental analyses of ${ }^{2} \mathrm{H}_{4}$-pitavastatin and RIF plasma concentration-time data were performed using Watson LIMS version 7.4.1 (Thermo Fisher Scientific Inc, Waltham, MA), which supported the generation of the various pharmacokinetic parameters; AUC, $t_{1 / 2}$ (half-life), CL (clearance), $V_{\mathrm{dss}}$ (volume of distribution), $C_{\max }$ (peak plasma concentration), and $t_{\max }$ (time to peak plasma concentration). As done for the different BAs, ${ }^{2} \mathrm{H}_{4}$-pitavastatin $\mathrm{AUCR}_{\text {plasma }}$ values were determined by dividing the $\mathrm{AUC}_{0-24 \text {,plasma }}$ after RIF treatment by the vehicle alone $\mathrm{AUC}_{0-24 \text {,plasma }}$.

For RIF, it was possible to calculate RIF plasma-free $C_{\max }$ based on a plasma unbound fraction of 0.265 (refer to Supplemental Material for RIF cynomolgus monkey plasma protein binding).

In turn, in vitro $\mathrm{IC}_{50}$ values for RIF with cynomolgus monkey OATP and NTCP (Shen et al., 2013; Chu et al., 2015) were used to calculate \% inhibition; $\%$ inhibition $=100 *\left\{[\mathrm{I}] /\left([\mathrm{I}]+\mathrm{IC}_{50}\right)\right\}$. It is assumed that $\mathrm{IC}_{50} \sim K_{\mathrm{i}}$ (when substrate concentration $<K_{\mathrm{m}}$ ). [I] represents plasma $C_{\max }$ of RIF (total or free). Consideration of free and total plasma $C_{\max }$ is consistent with Vaidyanathan et al. (2016); in the absence of i.v. RIF pharmacokinetic data, it was not possible to derive an absorption rate constant for RIF and estimate its liver inlet (portal) concentration in the cynomolgus monkey.

\section{Statistical Analysis of RIF Dose Response}

Plasma. Plasma profiles over time for each animal were collapsed into an AUC using the trapezoidal rule and a $C_{\max }$ score. The analyses were then conducted using a linear mixed model, such that the within-animal correlations were accounted for in the model. The $R$ computing language was used for these calculations (R Foundation for Statistical Computing, Vienna, Austria. http:// www.R-project.org/). Outcomes were analyzed on the $\log$ (base 2) scale to make
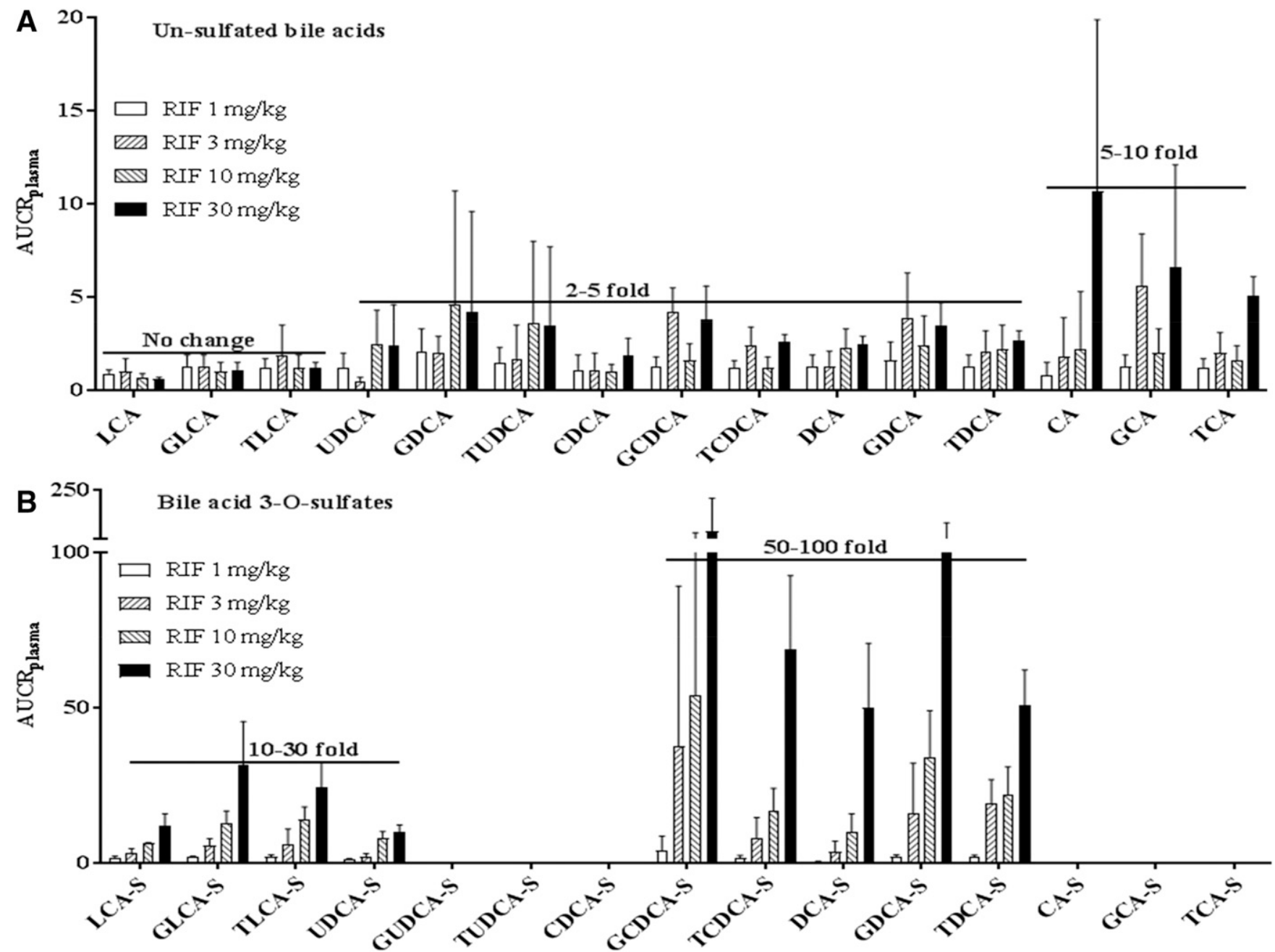

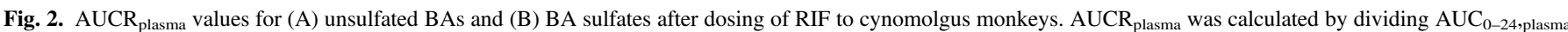

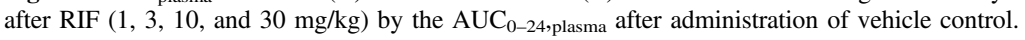


the residuals more normal. Concentrations were entered into the model on the same scale, and the $P$ value for the linear trend was based on an $F$-statistic using Satterthwaite's approximation (West et al., 2015). False discovery rate (FDR) estimates were computed using the Benjamini and Hochberg procedure (Benjamini and Hochberg, 1995).

Urine. Renal clearance $\left(\mathrm{CL}_{\text {renal }}\right)$ values $(\mathrm{ml} / \mathrm{min} / \mathrm{kg})$ of zero were treated as missing, and analytes with greater than five missing values were excluded. As such, 18 of the 30 BAs were analyzed. As described for plasma, the analysis was conducted using a linear mixed model such that the within-animal correlations were accounted for in the model. The BAs were analyzed on the natural $\log$ scale to make the residuals more normal. Similar to plasma, the $P$ value for the linear trend was based on an F-statistic using Satterthwaite's approximation and FDR estimates were computed using the Benjamini and Hochberg procedure.

\section{Results}

Pharmacokinetics of RIF in Cynomolgus Monkeys. As expected, a dose-dependent increase in $\mathrm{RIF} \mathrm{AUC}_{0-24 \text {,plasma }}$ and plasma $C_{\max }$ was observed over the dose range of $1-30 \mathrm{mg} / \mathrm{kg}$ (Table 1; Supplemental Fig. 1); however, at RIF doses of 3, 10, and $30 \mathrm{mg} / \mathrm{kg}$, there was evidence for a greater than proportional increase in $\mathrm{AUC}_{0-24, \text { plasma }}$ (11-, 71- and 243-fold versus $\mathrm{AUC}$ at $1 \mathrm{mg} / \mathrm{kg}$ ) and plasma $\mathrm{C}_{\max }$ (12-, 45-, and 137-fold versus $C_{\max }$ at $1 \mathrm{mg} / \mathrm{kg}$ ). For RIF, plasma unbound fraction was 0.265 and so the calculated free $C_{\max }$ was $0.06,0.66,2.57$, and $7.79 \mu \mathrm{M}$ at $1,3,10$, and $30 \mathrm{mg} / \mathrm{kg}$, respectively. Such concentrations exceed the reported in vitro $\mathrm{IC}_{50}$ values $(0.14-1.7 \mu \mathrm{M})$ for $\mathrm{RIF}$ with cynomolgus monkey OATP1B1 and OATP1B3 (Shen et al., 2013; Chu et al., 2015).
Impact of RIF on ${ }^{\mathbf{2}} \mathbf{H}_{\mathbf{4}}$-Pitavastatin Pharmacokinetics. The pharmacokinetic parameters of ${ }^{2} \mathrm{H}_{4}$-pitavastatin, an accepted cynomolgus monkey OATP probe drug (Takahashi et al., 2013), were determined after dosing of an i.v. bolus $(0.2 \mathrm{mg} / \mathrm{kg})$ to vehicle control and RIF-dosed cynomolgus monkeys (Table 2, Supplemental Fig. 1). Compared with the vehicle control, ${ }^{2} \mathrm{H}_{4}$-pitavastatin $\mathrm{CL}$ was decreased $21 \%, 58 \%, 73 \%$, and $76 \%$ with RIF treatment at $1,3,10$, and $30 \mathrm{mg} / \mathrm{kg}$ dose levels, respectively; however, the change was only statistically significant at the three top RIF doses. Furthermore, ${ }^{2} \mathrm{H}_{4}$-pitavastatin $\mathrm{AUC}_{0-24 \text {,plasma }}$ was increased 1.2-, 2.4-, 3.8-, and 4.5-fold at 1, 3, 10 and $30 \mathrm{mg} / \mathrm{kg} \mathrm{RIF}$, respectively. Both the $\mathrm{V}_{\mathrm{dss}}$ and $\mathrm{T}_{1 / 2}$ of ${ }^{2} \mathrm{H}_{4}$-pitavastatin were decreased $(\sim 70 \%)$ at the three highest RIF doses. At all the RIF dose levels, recovery of unchanged ${ }^{2} \mathrm{H}_{4}$-pitavastatin in urine was less than $5 \%$ of the dose. Overall, the impact of the lowest RIF dose on ${ }^{2} \mathrm{H}_{4}$-pitavastatin pharmacokinetics was not statistically significant.

Profiling of Plasma BAs at Different Doses of RIF. As with ${ }^{2} \mathrm{H}_{4}$ pitavastatin, the plasma levels of various BAs, as well as their corresponding 3-O-sulfate conjugates, were measured in cynomolgus monkeys after increasing doses of RIF. In total, 30 different BAs were monitored. Sulfated BAs, in particular GDCA-S, TDCA-S, GCDCA-S, TCDCA-S, GLCA-S, and TLCA-S, presented a marked dose-dependent increase in their plasma concentration-time profile (Fig. 1; Supplemental Table 3). In contrast, the plasma concentration-time profile of nonsulfated BAs, particularly DCA, GDCA, TDCA, GCDCA, TCDCA, UDCA, GUDCA, TUDCA, CA, GCA, and TCA, showed a relatively weak increase at the RIF doses of 10 and $30 \mathrm{mg} / \mathrm{kg}$ (Supplemental Fig. 2).

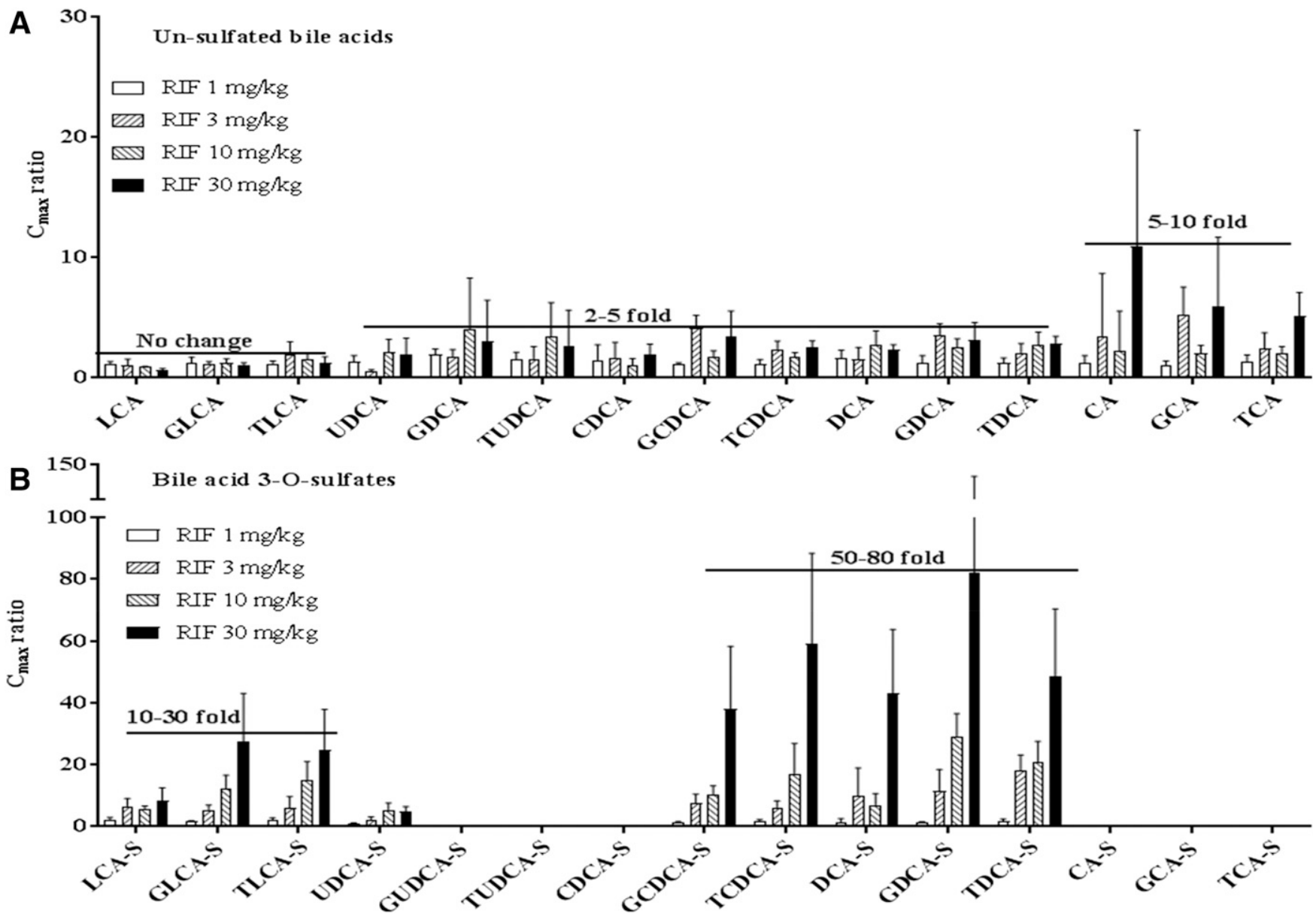

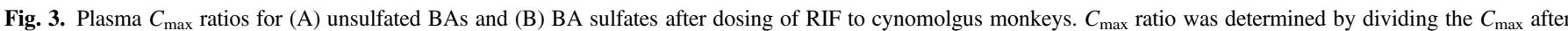
each RIF treatment by the $C_{\max }$ after administration of vehicle control. 
Chu et al. (2015) have also reported a weak increase in nonsulfated BAs after an oral RIF dose of $18 \mathrm{mg} / \mathrm{kg}$. RIF treatment did not cause significant changes in LCA, GLCA, and TLCA. Unfortunately, GUDCA-S, TUDCA-S, CA-S, TCA-S, and GCA-S plasma levels were low and remained undetectable, even after RIF treatment. Overall, the presence of various sulfo-conjugates in plasma was indicative of BA sulfation in the cynomolgus monkey. The pool of cynomolgus monkey BAs in circulation was distinct from that reported for human subjects; however, the percentages of sulfated $(1.3 \%$ versus $28.4 \%)$ and amidated $(21.5 \%$ versus $75.4 \%$ ) BAs were low for monkey versus human, respectively (Supplemental Table 4).

To complement the in vivo studies, the sulfation of six representative BAs (GCDCA, GUDCA, LCA, GLCA, GCA, and TLCA) was investigated after incubation with cynomolgus monkey liver cytosol (Supplemental Fig. 3); the availability of authentic standards supported identification of the 3-O-sulfate as the major product of PAPS-fortified monkey liver cytosol. Although a good correlation was obtained between human and cynomolgus monkey $\left(R^{2}=0.905\right)$, a low activity ratio (cynomolgus monkey-to-human) was obtained for the formation of LCA-S, GLCA-S, TLCA-S, and GUDCA-S (0.20, 0.25, 0.36, and 0.37, respectively). In comparison, GCDCA-S (activity ratio $=0.89$ ) and GCA-S (activity ratio $=3.0$ ) rendered higher activity ratios comparable to those of DHEA 3-O-sulfate (activity ratio of 1.3). Importantly, RIF (up to $100 \mu \mathrm{M}$ ) was shown not to inhibit cynomolgus monkey liver cytosol-catalyzed BA sulfation (Supplemental Fig. 4).

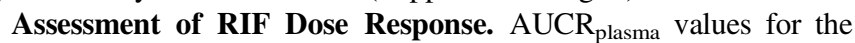
various BAs after RIF treatment are shown in Fig. 2. The highest AUCR $_{\text {plasma }}(\geq 78$ ) was observed for GDCA-S and GCDCA-S, followed by TDCA-S, DCA-S, and TCDCA-S ( 50) and GLCA-S and TLCA-S ( 30). A relatively modest AUCR plasma $(5-10)$ was observed for CA, GCA, and TCA. The remaining nonsulfated BAs presented

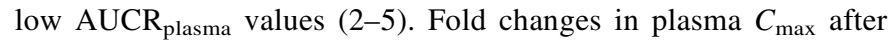
RIF treatment are shown in Fig. 3. Similar to the AUC changes, the highest (50- to 80-) fold increase was observed for DCA-S, GDCA-S, TCDCA-S, TDCA-S, and GCDCA-S, followed by a 20-fold increase for

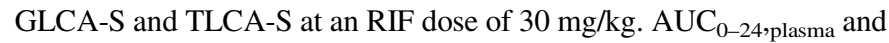
plasma $C_{\max }$ values at each RIF dose and vehicle control are shown in Supplemental Table 3.

Statistical analysis for linear trend in $\mathrm{AUC}_{0-24 \text {,plasma }}$ and $C_{\max }$ and FDR for each BA are shown in Table 3. Overall, with the exception of CA-S, all BA 3-O-sulfates were characterized by a significant linear trend $(P<0.01)$ with a slope of more than 0.6700 and an FDR less than $10 \%$ for AUC $_{0-24}$,plasma. GDCA-S, GCDCA-S, TDCA-S, TCDCA-S, GLCA-S, and TLCA-S were the most significant sulfate conjugates, with a $P$ value for the linear trend of less than 0.001 , a slope of more than 0.7500 , and FDR less than $1 \%$ for $\mathrm{AUC}_{0-24}$,plasma, as well as $C_{\max }$. In contrast, most of the nonsulfated bile acids did not show a statistically significant linear trend, and FDR was more than $10 \%$ for $\mathrm{AUC}_{0-24 \text {,plasma }}$ and $C_{\max }$. Only TCA and TDCA showed a $P$ value less than 0.01 and $\sim 10 \%$ FDR.

Importantly, the plasma $\mathrm{AUCR}_{\text {plasma }}$ values showed a good linear correlation $\left(R^{2}>0.7000\right)$ between pitavastatin and GDCA-S, TDCA-S, GCDCA-S, TCDCA-S, GLCA-S, and TLCA-S (Fig. 4). Similarly, a good linear correlation $\left(R^{2}>0.7500\right)$ between RIF plasma-free $C_{\max }$ and ${ }^{2} \mathrm{H}_{4}$-pitavastatin, GDCA-S, TDCA-S, GCDCA-S, TCDCA-S, GLCA-S, and TLCA-S AUCR plasma was observed (Fig. 5).

TABLE 3

$\mathrm{P}$ value for the linear trend with RIF dose and FDR estimates for the area under the plasma concentration-time curve $\left(\mathrm{AUC}_{0-24 \text {,plasma }}\right)$, maximum plasma concentration $\left(C_{\text {max,plasma }}\right)$ and renal clearance $\left(\mathrm{CL}_{\text {renal }}\right)$ for various $\mathrm{BAs}$ and their respective 3-O-sulfate conjugates

\begin{tabular}{|c|c|c|c|c|c|c|c|c|c|}
\hline \multirow{2}{*}{$P$ value } & \multicolumn{3}{|c|}{$\mathrm{AUC}_{0-24}$,plasma } & \multicolumn{3}{|c|}{$\mathrm{C}_{\text {max,plasma }}$} & \multicolumn{3}{|c|}{$\mathrm{CL}_{\text {renal }}$} \\
\hline & Slope ${ }^{a}$ & $P$ Value & FDR & Slope & $P$ Value & FDR & Slope & $P$ Value & FDR \\
\hline CA-S & 2.08 & 0.0218 & 0.2395 & 1.06 & 0.0589 & 0.5889 & $\mathrm{NA}^{b}$ & NA & NA \\
\hline DCA-S $S^{c}$ & 1.44 & $<0.0001$ & $<0.0001$ & 0.90 & $<0.0001$ & $<0.0001$ & -0.005 & 0.7980 & 1.0000 \\
\hline TCA-S & 1.2 & 0.0004 & 0.0067 & 1.07 & 0.0011 & 0.0193 & NA & NA & NA \\
\hline GDCA-S $^{c}$ & 1.13 & $<0.0001$ & $<0.0001$ & 1.11 & $<0.0001$ & 0.0001 & 0.003 & 0.7670 & 1.0000 \\
\hline GCA-S & 1.1 & 0.0021 & 0.0373 & 0.89 & 0.0067 & 0.1067 & NA & NA & NA \\
\hline TCDCA-S ${ }^{c}$ & 1.09 & $<0.0001$ & $<0.0001$ & 1.04 & $<0.0001$ & $<0.0001$ & 0.010 & 0.2517 & 1.0000 \\
\hline GCDCA-S ${ }^{c}$ & 1.07 & $<0.0001$ & $<0.0001$ & 0.97 & $<0.0001$ & $<0.0001$ & -0.020 & 0.3157 & 1.0000 \\
\hline CDCA-S & 0.99 & 0.0036 & 0.0619 & 0.61 & 0.0940 & 0.6907 & NA & NA & NA \\
\hline $\mathrm{TDCA}^{-\mathrm{S}^{c}}$ & 0.91 & $<0.0001$ & $<0.0001$ & 0.89 & $<0.0001$ & 0.0001 & 0.010 & 0.4206 & 1.0000 \\
\hline GLCA-S ${ }^{c}$ & 0.81 & $<0.0001$ & $<0.0001$ & 0.78 & $<0.0001$ & $<0.0001$ & -0.003 & 0.7155 & 1.0000 \\
\hline TLCA-S $^{c}$ & 0.78 & $<0.0001$ & $<0.0001$ & 0.76 & $<0.0001$ & $<0.0001$ & -0.003 & 0.7002 & 1.0000 \\
\hline UDCA-S & 0.77 & $<0.0001$ & $<0.0001$ & 0.56 & $<0.0001$ & 0.0009 & 0.003 & 0.6382 & 1.0000 \\
\hline $\mathrm{CA}$ & 0.72 & 0.0049 & 0.0737 & 0.49 & 0.0251 & 0.3264 & 0.006 & 0.7236 & 1.0000 \\
\hline LCA-S & 0.68 & $<0.0001$ & $<0.0001$ & 0.35 & 0.0013 & 0.0219 & $N A^{\mathrm{b}}$ & NA & NA \\
\hline TCA & 0.35 & 0.0071 & 0.092 & 0.35 & 0.0006 & 0.012 & -0.030 & 0.0162 & 0.2434 \\
\hline GCA & 0.30 & 0.0971 & 0.8231 & 0.31 & 0.0767 & 0.6907 & -0.005 & 0.6641 & 1.0000 \\
\hline DCA & 0.25 & 0.0049 & 0.0737 & 0.18 & 0.0222 & 0.3107 & 0.040 & 0.0086 & 0.1369 \\
\hline UDCA & 0.24 & 0.0112 & 0.1343 & 0.19 & 0.0829 & 0.6907 & 0.040 & 0.004 & 0.0685 \\
\hline TDCA & 0.23 & 0.0045 & 0.0724 & 0.26 & 0.0004 & 0.0075 & 0.020 & 0.0485 & 0.6303 \\
\hline GDCA & 0.21 & 0.0622 & 0.6218 & 0.23 & 0.0346 & 0.4152 & 0.050 & 0.0027 & 0.0478 \\
\hline GCDCA & 0.17 & 0.2221 & 0.8884 & 0.18 & 0.1857 & 1.0000 & NA & NA & NA \\
\hline TCDCA & 0.16 & 0.0915 & 0.8231 & 0.19 & 0.0073 & 0.1088 & -0.030 & 0.0226 & 0.3161 \\
\hline CDCA & 0.16 & 0.1127 & 0.8231 & 0.09 & 0.3571 & 1.0000 & 0.003 & 0.7522 & 1.0000 \\
\hline TUDCA & 0.15 & 0.1343 & 0.8231 & 0.11 & 0.3341 & 1.0000 & NA & NA & NA \\
\hline GUDCA & 0.10 & 0.2289 & 0.8884 & 0.05 & 0.5432 & 1.0000 & NA & NA & NA \\
\hline GLCA & -0.06 & 0.4130 & 0.8884 & -0.03 & 0.669 & 1.0000 & NA & NA & NA \\
\hline LCA & -0.1 & 0.1097 & 0.8231 & -0.13 & 0.0434 & 0.4771 & -0.009 & 0.5259 & 1.0000 \\
\hline
\end{tabular}

NA, not applicable.

${ }^{a}$ Results ranked in terms of slope $\left(\mathrm{AUC}_{0-24, \text { plasma }}\right)$

${ }^{b}$ No baseline (vehicle control) levels; NA: not applicable.

${ }^{c}$ Considered candidate OATP biomarkers (See Fig. 2 and 3). 

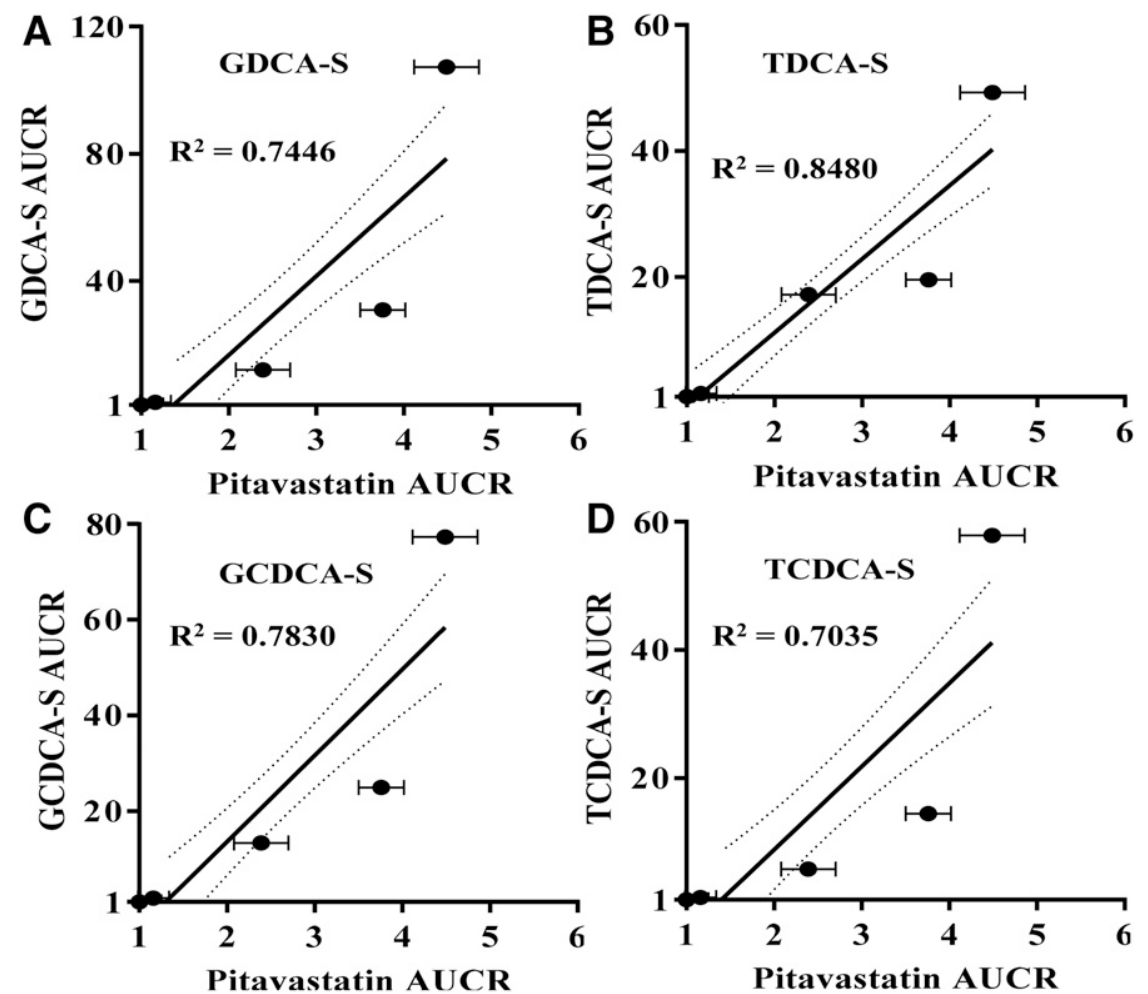

Fig. 4. Linear regression of $A_{U C R}$ plasma between ${ }^{2} \mathrm{H}_{4}$-pitavastatin and GDCA-S (A), TDCA-S (B), GCDCA-S (C), TCDCA-S (D), GLCA-S (E), and TLCA-S (F) in cynomolgus monkeys. AUCR plasma $_{\text {was determined as described }}$ in the legend to Fig. 2.
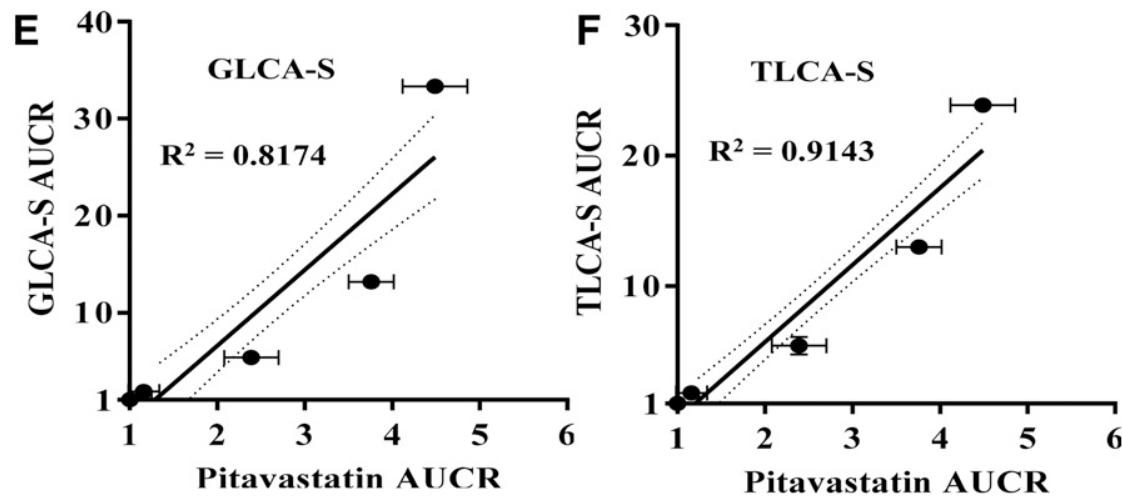

As described previously, an effort was made to administer RIF over a dose range that generated a wide range of plasma total $(0.2-29 \mu \mathrm{M})$ and free $(0.06-7.8 \mu \mathrm{M}) C_{\max }$ values (Table 1$)$. In so doing, it was possible to investigate the dose-dependent inhibition of OATPs and NTCP. Based on in vitro IC $_{50}$ data, dosedependent inhibition of OATP1B $1(16 \%$ to $\geq 96 \%)$ and OATP1B3 $(<10 \%-\geq 85 \%)$ was expected, with less inhibition of OATP2B1 $(<10 \%-31 \%)$ and NTCP $(<10 \%-29 \%)$ anticipated (Supplemental Table 5 and 6). Despite the effort to ensure dose-dependent inhibition, however, the $\mathrm{AUCR}_{\text {plasma }}$ values for the various BA sulfates differed markedly. The highest maximal AUCR $_{\text {plasma }}$ values $(\geq 78)$ were obtained with GDCA-S and GCDCA-S, followed by TDCA-S, DCA-S, and TCDCA-S (AUCR plasma $_{2}$ 50), GLCA-S and TLCA-S (AUCR plasma $~_{30}$ ).

Impact of RIF on BA Renal Clearance. Because the 3-O-sulfate conjugates of the various BAs are recovered in human urine (Bathena et al., 2013, 2015; Tsuruya et al., 2016), the present study was extended to include the profiling of urine of control and RIF-dosed cynomolgus monkeys. In this regard, a dose-dependent increase in the amounts of GDCA-S, TDCA-S, GCDCA-S, TCDCA-S, GLCA-S, and TLCA-S excreted in urine was observed (Supplemental Table 3). $\mathrm{CL}_{\text {renal }}$ ratios and statistical analysis for each BA are shown in Fig. 6 and Table 3, respectively. A weak dose-dependent increase in $\mathrm{CL}_{\text {renal }}$ was observed for UDCA, GDCA, and DCA; however, there was no statistically significant effect of RIF treatment on the $\mathrm{CL}_{\text {renal }}$ of sulfated and nonsulfated bile acids. For the former, this is in marked contrast to the changes in plasma AUC and $\mathrm{C}_{\max }$ after RIF treatment.

Incubation of GCDCA-S, GDCA-S, DCA-S, TDCA-S, TCA, and Pitavastatin with Cynomolgus Monkey Plated Hepatocytes. Based on the availability of deuterium-labeled material and RIF-dependent $\mathrm{AUCR}_{\text {plasma }}$ values in vivo, four sulfated BAs (GCDCA-S, GDCA-S, DCA-S, and TDCA-S) were chosen for study as solute carrier (SLC) substrates in vitro after incubation with plated cynomolgus monkey primary hepatocytes (Figs. 7 and 8). TCA and pitavastatin were also incubated as representative cynomolgus monkey NTCP (>OATP) and OATP ( $>$ NTCP) substrates, respectively (Chu et al., 2015; Takahashi et al., 2013). To discern the role of NTCP versus OATPs, incubations were performed in the presence and absence of sodium (NTCP is sodium-dependent). In addition, RIF (5 $\mu \mathrm{M})$ and RIFsv (1 $\mathrm{mM})$ were deployed as cynomolgus monkey OATP-selective (OATP1B1 and OATP1B3 inhibition $\geq 75 \%$; NTCP inhibition $\leq 13 \%$ ) and pan-SLC (OATP and NTCP $\geq 97 \%$ inhibition) 

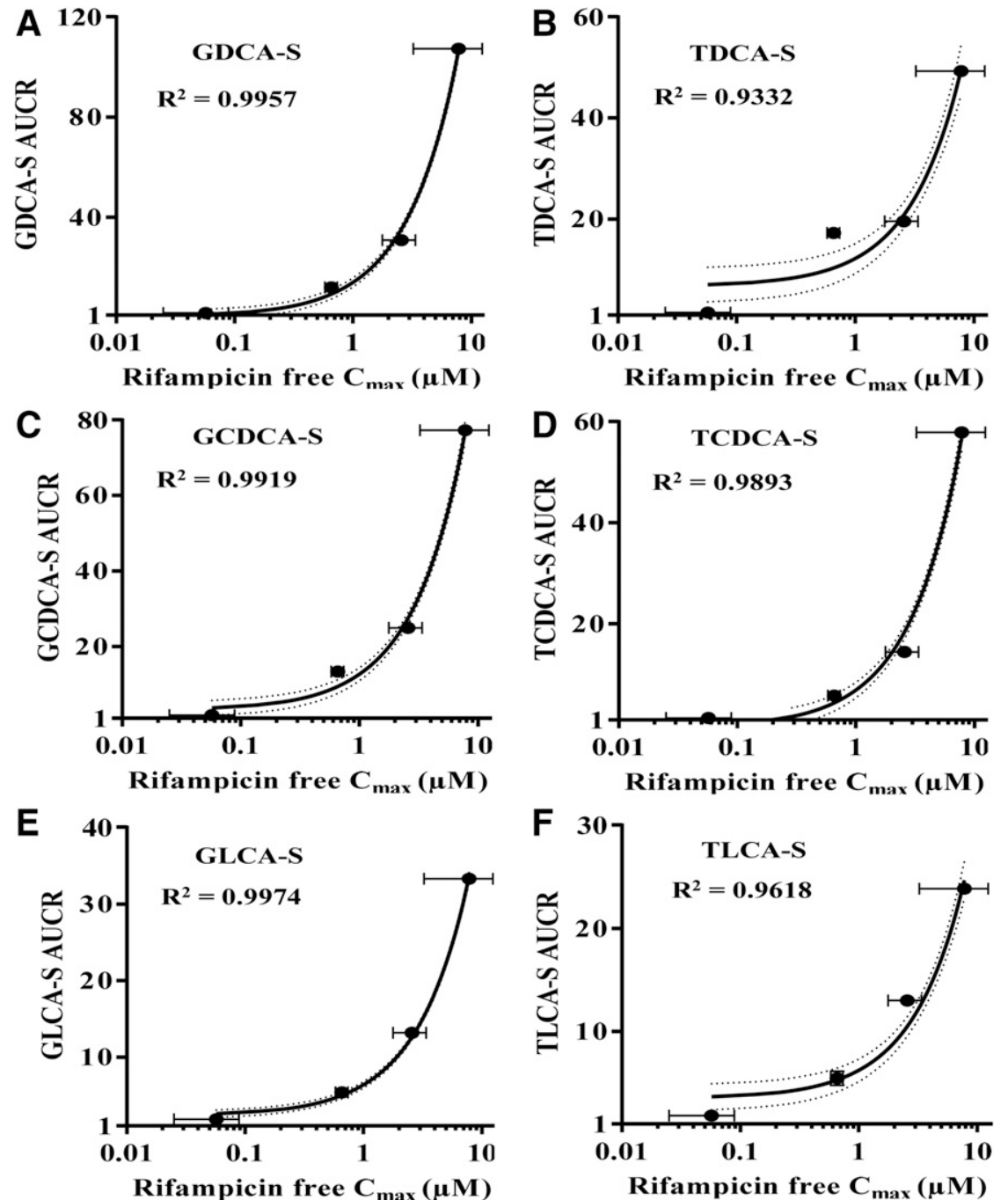

Fig. 5. Linear regression between cynomolgus monkey RIF plasma-free $C_{\max }(\mu \mathrm{M})$ at $1,3,10$, and $30 \mathrm{mg} / \mathrm{kg}$ and AUCR $_{\text {plasma }}$ for GDCA-S (A), TDCA-S (B), GCDCA-S (C), TCDCA-S (D), GLCA-S (E), TLCA-S (F), and ${ }^{2} \mathrm{H}_{4-}$ pitavastatin $(\mathrm{G})$. AUCR $\mathrm{Alasma}_{\text {was determined as described }}$ in the legend to Fig. 2.

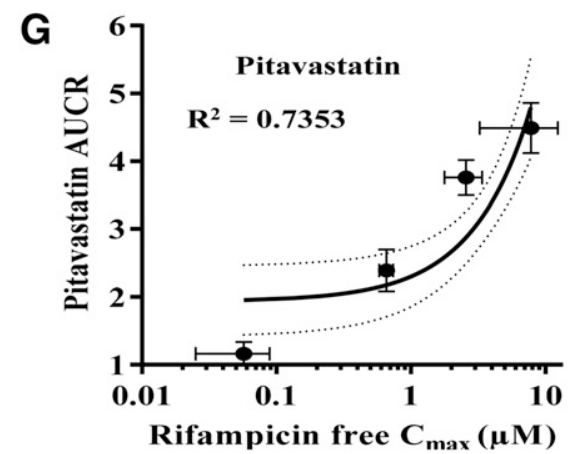

inhibitors, respectively (Supplemental Table 5) (Shen et al., 2013; Chu et al., 2015; Hong Shen, Bristol-Myers Squibb, personal communication).

When incubated with RIFsv, the uptake of TCA, GDCA-S, GCDCA-S, and pitavastatin was markedly inhibited ( $\geq 92 \%)$. For the four substrates, such a result is consistent with relatively high rates of active (versus passive; $\leq 8 \%$ ) uptake (Fig. 7A). As shown in Fig. 7B, the uptake of GCDCA-S and GDCA-S was minimally impacted by the removal of sodium. By contrast, uptake of TCA (74\%) and pitavastatin $(\sim 44 \%)$ was decreased in the presence of sodium-free buffer. As expected, RIF elicited relatively weak inhibition of TCA uptake compared with pitavastatin (14\% versus $58 \%)$. Uptake of both
GCDCA-S (69\% inhibition) and GDCA-S (82\% inhibition) was sensitive to RIF. Although the exact contribution of OATP1B1 and OATP1B3 was not determined, in the absence of selective inhibitors and established relative activity factors for cynomolgus monkey transporters, it is concluded that uptake of both GCDCA-S and GDCA-S $(0.5 \mu \mathrm{M})$ in the presence of cynomolgus monkey hepatocytes is dominated by OATPs and that their profile is distinct from that of TCA. Based on the results presented in Fig. 8, the same can be said for two additional BA sulfates (TDCA-S and DCA-S). In this instance, RIF $(5 \mu \mathrm{M})$ was shown to inhibit the uptake of TCA, pitavastatin, GCDCA-S, GDCA-S, TDCA-S, and DCA-S $(0.1 \mu \mathrm{M})$ by $21 \%, 73 \%, 92 \%, 83 \%, 95 \%$, and $80 \%$, respectively. 

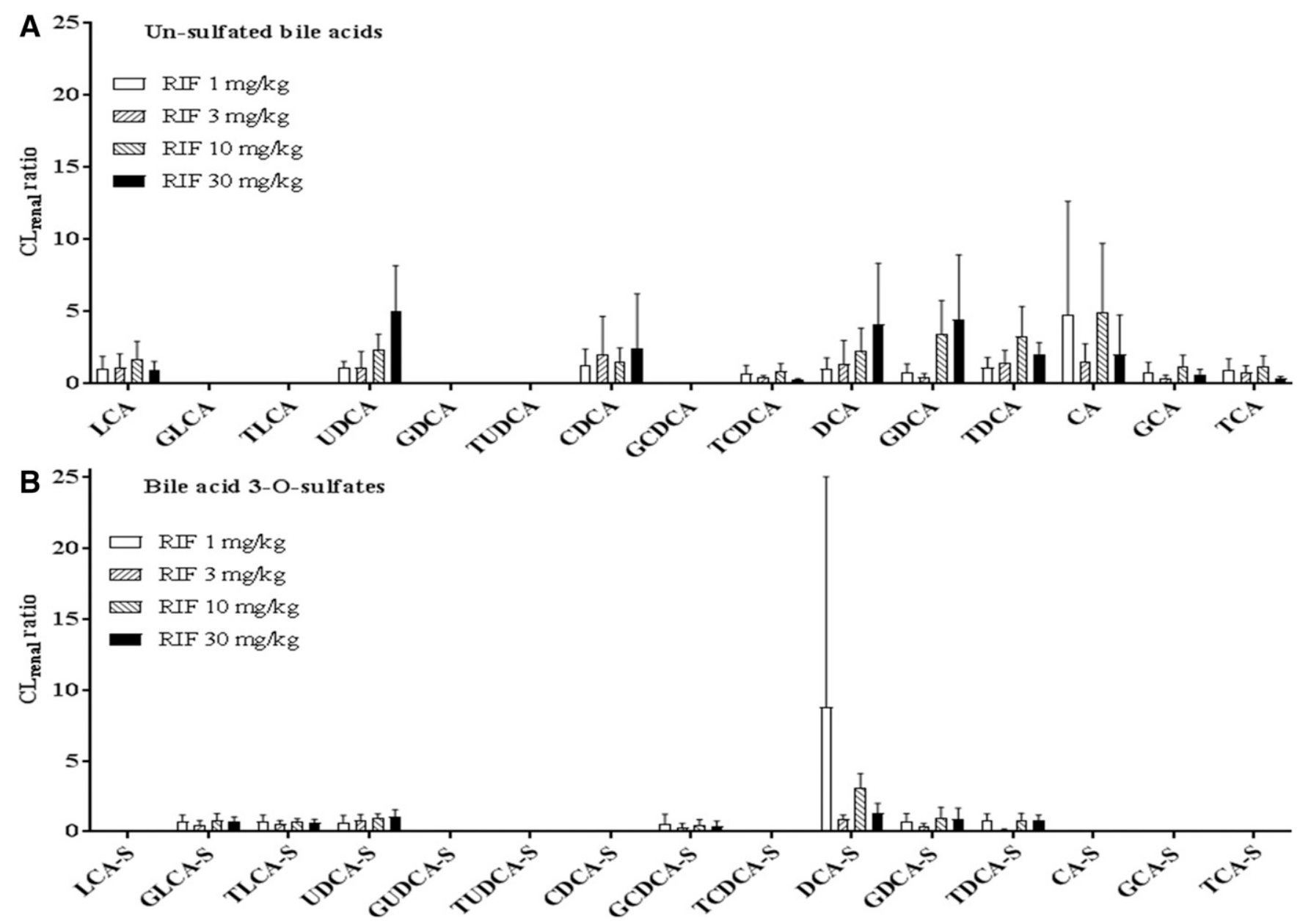

Fig. 6. Cynomolgus monkey $\mathrm{CL}_{\text {renal }}$ ratios for (A) unsulfated BAs and (B) BA sulfates at increasing doses of RIF. $\mathrm{CL}_{\text {renal }}$ ratio determined by dividing the $\mathrm{CL}_{\text {renal }}$ after RIF treatment by the $\mathrm{CL}_{\text {renal }}$ after vehicle alone.

\section{Discussion}

Metabolism of various BAs is complex and involves oxidation, amidation, glucuronidation, and sulfation. Once conjugated, BAs are also subjected to transporter-mediated uptake (e.g., OATP and NTCP) and efflux (Akita et al., 2001; Sasaki et al., 2002; Zelcer et al., 2003a, 2003b; Tsuruya et al., 2016; Rodrigues et al., 2014). Therefore, the BA pool of most species is complex and subject to enterohepatic recirculation and renal clearance. This means that BA profiling requires robust LC-MS/MS methods with access to a large number of authentic standards (Bathena et al., 2013). To support the present study, therefore, authentic standards of a number of noncommercially available BA 3-O-sulfates were prepared, and 30 different BAs were profiled in cynomolgus monkey plasma and urine.

Although in vivo sulfation of DHEA has been reported in cynomolgus monkeys (Leblanc et al., 2003), and SULT2A1 (human sulfotransferase involved in BA 3-O-sulfation) is known to be expressed in cynomolgus monkey liver (Nishimura et al., 2008; Alnouti, 2009; Nishimura et al., 2009), there have been no reports describing the BA sulfation in the cynomolgus monkey. For the first time, it was possible to report that DHEA and various BAs undergo sulfation in vitro (Supplemental Fig. 3 ). Importantly, the presence of BA sulfates in cynomolgus monkey urine is consistent with human data (Bathena et al., 2013; Tsuruya et al., 2016); however, the fraction of the BA pool in circulation as the sulfated species was low in cynomolgus monkey versus human $(28.4 \%$ versus 1.3\%; Supplemental Table 4) and likely reflects species differences in
$\mathrm{CL}_{\text {renal }}$ and formation clearance. In agreement, the $\mathrm{CL}_{\text {renal }}$ of GCDCA-S is lower in humans (31 versus $0.05 \mathrm{ml} / \mathrm{min}$ per kilogram) (Supplemental Table 3; Tsuruya et al., 2016), and the rate of BA sulfation in vitro was lower in the presence of cynomolgus monkey cytosol (Supplemental Fig. 3). A species difference in OATP-mediated hepatic uptake clearance is also a possibility.

GCDCA-S has been shown to undergo active renal secretion and has been proposed as a biomarker for human organic anion transporter 3 (OAT3) (Tsuruya et al., 2016). Although cynomolgus monkey OAT3 has been expressed and characterized, nothing is known of its ability to transport BA sulfates and its inhibition by RIF (Tahara et al., 2005). Therefore, as part of the present study, it was important to assess the impact of RIF on BA sulfation and renal clearance. This ensured that any

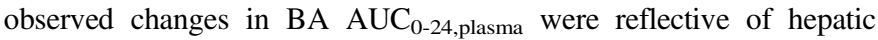
transporter inhibition. In vitro data indicated that RIF (up to $100 \mu \mathrm{M}$ ) did not inhibit liver cytosol-catalyzed BA sulfation (Supplemental Fig. 4). Likewise, profiling of cynomolgus monkey urine supported calculation of $\mathrm{CL}_{\text {renal }}$ for the different BAs and it was determined that the impact of RIF was minimal (Table 3; Supplemental Table 3). Importantly, there is evidence indicating that BA sulfates also serve as substrates of human canalicular multidrug resistance-associated protein (MRP) MRP2 and basolateral MRP3 and MRP4 (Akita et al., 2001; Sasaki et al., 2002; Zelcer et al., 2003a, 2003b). The possibility that single dose RIF can inhibit cynomolgus monkey MRP2 has already been considered by Chu et al. (2015). In this instance, the authors showed that RIF is a relatively 

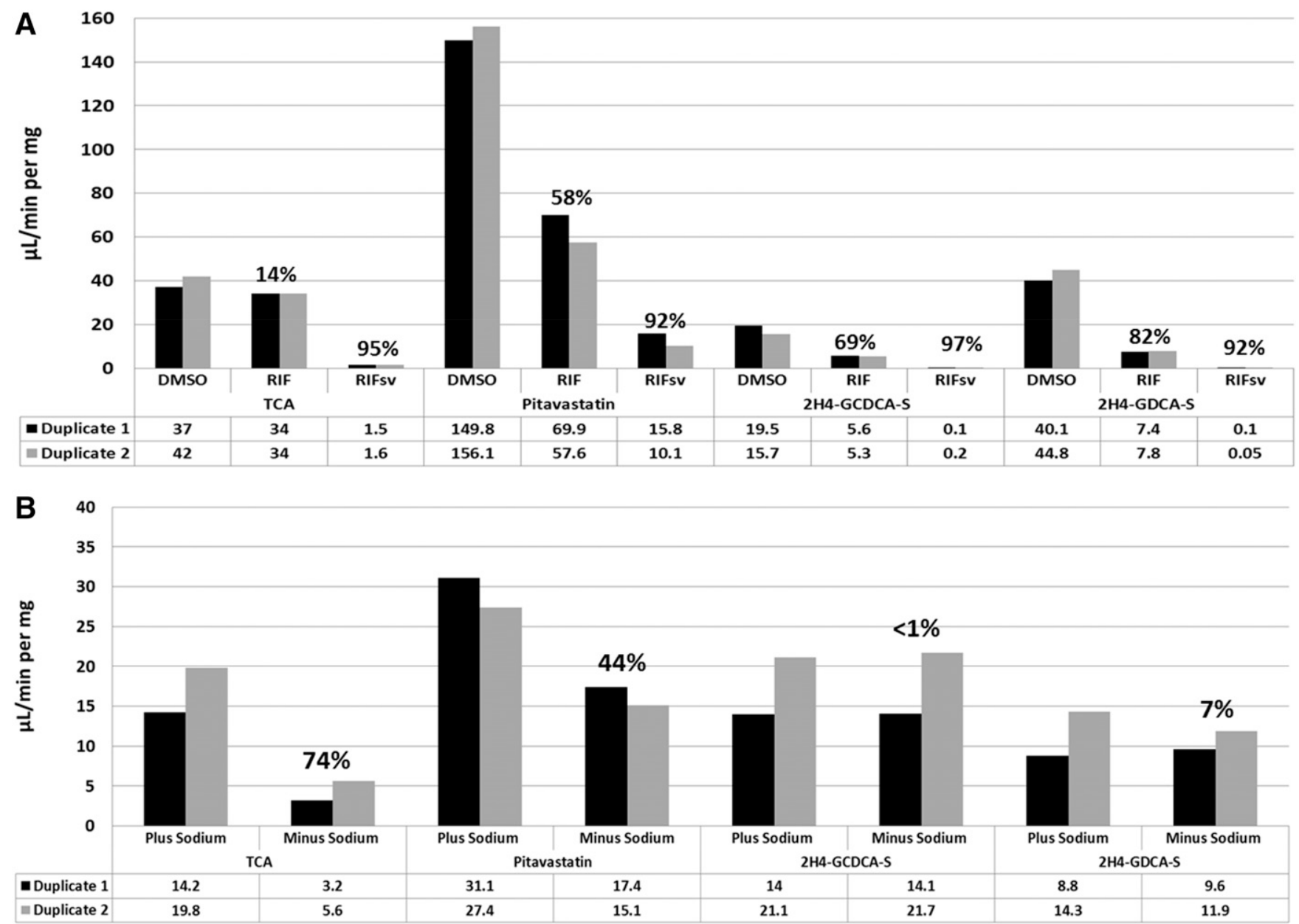

Fig. 7. Impact of RIF and RIFsv (A) and sodium removal (B) on the uptake of ${ }^{2} \mathrm{H}_{5}$-GCDCA-S $(0.5 \mu \mathrm{M}),{ }^{2} \mathrm{H}_{4}$-GDCA-S $(0.5 \mu \mathrm{M}),{ }^{2} \mathrm{H}_{4}$-TCA (0.5 $\left.\mu \mathrm{M}\right)$, and non-labeled pitavastatin $(0.1 \mu \mathrm{M})$ by plated cynomolgus monkey primary hepatocytes. RIF and RIFsv were added at a final concentration of $5 \mu \mathrm{M}$ and $1 \mathrm{mM}$, respectively. Data for individual duplicates are shown. The mean $\%$ decrease is shown also and calculated as $\left(\frac{v_{D M S O}-v_{i}}{v_{\text {DMSO }}}\right) * 100 . v_{\mathrm{DMSO}}$ and $v_{\mathrm{i}}$ is the mean uptake rate in the presence of DMSO alone (plus sodium) and inhibitor (or minus sodium) respectively.

weak inhibitor of cynomolgus monkey $\mathrm{MRP} 2\left(\mathrm{IC}_{50}=118 \mu \mathrm{M}\right)$ and argued for relatively minimal inhibition based on estimated free RIF liver levels $(\sim 10 \mu \mathrm{M})$. Although no RIF inhibition data are available for monkey MRP3 and MRP4, given the robust $\mathrm{AUCR}_{\text {plasma }}$ values for the various BA sulfates, it is assumed that neither transporter is inhibited. Alternatively, RIF could induce MRP3 and MRP4 and compromise interpretation of the data (Marschall et al., 2005; Badolo et al., 2015); however, it can be argued that significant and sustained induction of both MRP proteins is unlikely after a single RIF dose. Importantly, BA sulfate plasma concentration-time profiles were consistent with relatively rapid (within 6 hours) dose-dependent inhibition of hepatic uptake. Moreover, plasma concentrations for five of the six BA sulfates were trending toward pre-RIF dose levels by 24 hours (Fig. 1).

To date, efforts to inhibit cynomolgus monkey OATP in vivo have involved oral administration of a single RIF dose that generates a plasma total $C_{\max }$ of $\sim 10 \mu \mathrm{M}$ (Shen et al., 2013; Takahashi et al., 2013; Chu et al., 2015). As described, it was possible to administer RIF at four dose levels of $1,3,10$, and $30 \mathrm{mg} / \mathrm{kg}$ and obtain mean plasma total $C_{\max }$ values of $0.2,2.5,9.7$, and $29 \mu \mathrm{M}$, respectively; corresponding to a plasma-free $C_{\max }$ of $0.06,0.66,2.57$, and $7.79 \mu \mathrm{M}$, respectively (Table 1). Evidently, the pharmacokinetic profile of RIF in the cynomolgus monkey was nonlinear, likely reflecting saturation of first pass and consistent with human data (Acocella, 1978). Despite the nonlinearity, there was a good linear correlation $\left(R^{2}>0.9332\right)$ between

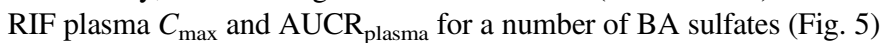
and, because expression of hepatic OATP2B1 is relatively low in cynomolgus monkey, such a dose response is more likely reflective of OATP1B1 and OATP1B3 inhibition (Wang et al., 2015). Based on the in vitro $\mathrm{IC}_{50}$ values reported for both OATPs $(\leq 1.7 \mu \mathrm{M}$; Supplemental Table 5), and assuming that RIF plasma concentrations support estimates of inhibition (Vaidyanathan et al., 2016), up to $>85 \%$ (RIF-free $C_{\max }$ ) and $>96 \%$ (RIF total $C_{\max }$ ) inhibition is anticipated (Supplemental Table 6). In agreement, a clear RIF dose-dependent increase in ${ }^{2} \mathrm{H}_{4}$-pitavastatin $\mathrm{AUCR}_{\text {plasma }}(1.2,2.4,3.8$, and 4.5) was evident (Fig. 5). Because of weaker inhibition by RIF ( IC $_{50} \geq 35.1 \mu \mathrm{M}$, Supplemental Table 5), less NTCP inhibition (10\%-29\%) is expected (Supplemental Table 6). This is an important consideration because certain BAs are known to favor NTCP over OATPs (Meier et al., 1997; Maeda et al., 2006; Dong et al., 2015: Suga et al., 2017). For example, the uptake of TCA by primary cynomolgus monkey hepatocytes was shown to be highly sodium dependent and relatively refractory to RIF (Figs. 7 and 8). Although no formal in vitro-in vivo exercise was attempted, it was assumed that the RIF dose-dependent $\mathrm{AUCR}_{\text {plasma }}$ values for TCA $(1.2,2.0,1.8$, and 5.4) are largely reflective of NTCP inhibition (Supplemental Tables 3 and 6). On the other hand, GCDCA-S, GDCA-S, TDCA-S, and DCA-S behaved as 


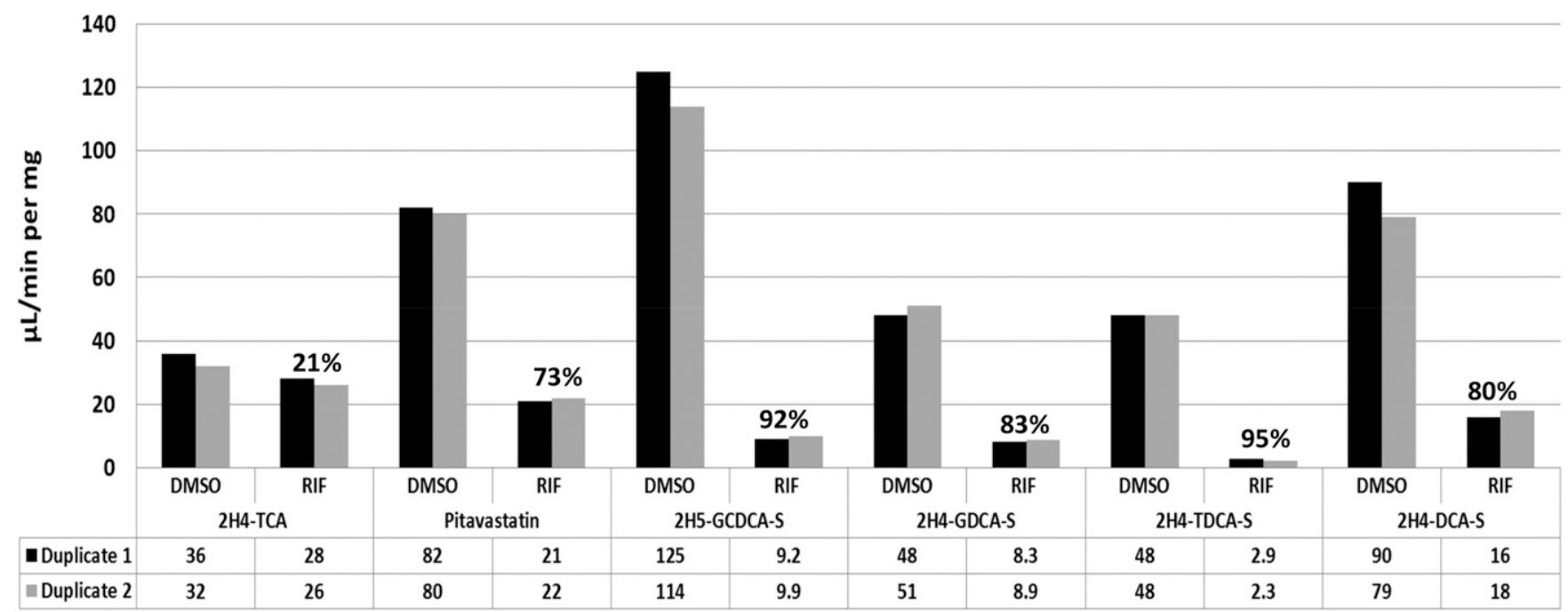

Fig. 8. Impact of RIF on the uptake of ${ }^{2} \mathrm{H}_{5}$-GCDCA-S $(0.1 \mu \mathrm{M}),{ }^{2} \mathrm{H}_{4}$-GDCA-S $(0.1 \mu \mathrm{M}),{ }^{2} \mathrm{H}_{4}$-TCA $(0.1 \mu \mathrm{M}),{ }^{2} \mathrm{H}_{4}$-TDCA-S $(0.1 \mu \mathrm{M}),{ }^{2} \mathrm{H}_{4}$-DCA-S $(0.1 \mu \mathrm{M})$, and nonlabeled pitavastatin $(0.1 \mu \mathrm{M})$ by plated cynomolgus monkey primary hepatocytes. RIF was added at a final concentration of $5 \mu \mathrm{M}$. Data for individual duplicates are shown. The mean \% decrease is shown and was calculated as described in the legend to Fig 7.

OATP substrates in the presence of cynomolgus monkey hepatocytes (Figs. 7 and 8), consistent with reports of a fifth sulfated BA (TLCA-S) presenting as an OATP substrate in vitro (Meng et al., 2002; Sasaki et al., 2002).

As described, five sulfated BAs in plasma responded robustly to RIF in a dose-dependent manner (maximal $\mathrm{AUCR}_{\text {plasma }} \geq 50$ ). Two additional BA sulfates, GLCA-S and TLCA-S, were also found to respond to RIF (maximal AUCR plasma $_{2}$ 30) (Fig. 2). From the standpoint of detectability in control animals, the magnitude of the RIF dose response, and detectability in human plasma (Supplemental Table 4), these seven BA sulfates (GCDCA-S, TCDCA-S, DCA-S, GDCA-S, TDCA-S, GLCA-S and TLCA-S) all present as potential sensitive OATP biomarkers. Importantly, the RIF dose response obtained was far greater than the increases $(<10$-fold) reported for cynomolgus monkey plasma bilirubin, bilirubin glucuronide, coproporphyrin (I and III), nonsulfated BAs, and DHEA sulfate (Chu et al., 2015; Watanabe et al., 2015; Shen et al., 2016).

Although the results presented herein showcase various BA sulfates as sensitive cynomolgus monkey OATP biomarkers, it cannot be assumed that the results translate directly to human subjects. For example, the balance of OATP- versus NTCP-mediated liver uptake and the contributions of individual OATPs may not be the same across species. Despite the caveats, the results of the present work are consistent with plasma metabolomic data from an OATP1B1 (SLCO1B1) genome-wide association study that identified GCDCA-S, GDCA-S, and TLCA-S as potential OATP1B1 biomarkers (Yee et al., 2016). Such BA sulfates could potentially serve as sensitive human OATP biomarkers and provide the necessary dynamic range to support perpetrator differentiation (weak, moderate, versus potent inhibition), enable the study of OATP genotype-phenotype associations, and facilitate phenotyping of hepatobiliary diseased subjects (Gong and Kim, 2013; Clarke et al., 2014). In this regard, sulfated BAs could be superior human OATP biomarkers compared with plasma coproporphyrin I and III (AUCR plasma 4.0) (Lai et al., 2016). As discussed previously, because circulating sulfated BAs are substrates of renal transporters also, they may serve as dual liver OATP and renal OAT3 biomarkers. In agreement, an increase in "urinary sulfated bile acids" has been reported in patients with various hepatobiliary diseases, which may in part reflect altered liver OATP function (Kobayashi et al., 2002; Nanashima et al., 2009; Bathena et al., 2015). Consistent with human data, the amount of sulfated BAs in cynomolgus monkey urine increased with RIF dose (Supplemental Table 3).

Based on the results of the present study, it is concluded that the cynomolgus monkey can form BA sulfates that comprise only $\sim 1 \%$ of the plasma BA pool and present as sensitive hepatic OATP biomarkers. Given the number of similarly sulfated BAs that are detectable in human serum (Bathena et al., 2013), it is envisioned that they will be increasingly studied as human OATP substrates and compared with other biomarkers such as coproporphyrin I and III.

\section{Acknowledgments}

The authors thank Dr. Hong Shen (Bristol-Myers Squibb Co., Princeton, NJ) for providing rifamycin SV in vitro inhibition data for cynomolgus monkey OATPs and NTCP expressed in HEK293 cells (Supplemental Table 5); Dana Gates and Sweta Modi (Pfizer Inc.) for help preparing the ${ }^{2} \mathrm{H}_{4}$-pitavastatin and rifampicin dose formulations; John Deschenes (Pfizer Inc.) for help conducting the cynomolgus monkey pharmacokinetics study; Sangwoo Ryu and Keith Riccardi (Pfizer Inc.) for conducting equilibrium dialysis of RIF with cynomolgus monkey plasma (Supplemental Material); and Brian Rago (Pfizer Inc.) for conducting bioanalysis of the cynomolgus monkey hepatocyte incubates.

\section{Authorship Contributions}

Participated in research design: Rodrigues, Gao, Varma, Tremaine, Thakare, Kosa.

Conducted experiments: Thakare, Gao, Kosa, Bi, Cerny, Sharma, Walker, Niosi.

Contributed new reagents and analytical procedures: Thakare, Alnouti, Huang, Liu., Yu, Walker.

Performed data analysis: Thakare, Varma, Bi, Kosa Kuhn, Rodrigues, Niosi. Wrote or contributed to the writing of the manuscript: Thakare, Gao, Kosa, Bi, Cerny, Sharma, Kuhn, Alnuti, Varma, Rodrigues.

\section{References}

Acocella G (1978) Clinical pharmacokinetics of rifampicin. Clin Pharmacokinet 3:108-127. Akita H, Suzuki H, Ito K, Kinoshita S, Sato N, Takikawa H, and Sugiyama Y (2001) Characterization of bile acid transport mediated by multidrug resistance associated protein 2 and bile salt export pump. Biochim Biophys Acta 1511:7-16.

Alnouti Y (2009) Bile acid sulfation: a pathway of bile acid elimination and detoxification. Toxicol Sci 108:225-246.

Badolo L, Jensen B, Säll C, Norinder U, Kallunki P, and Montanari D (2015) Evaluation of 309 molecules as inducers of CYP3A4, CYP2B6, CYP1A2, OATP1B1, OCT1, MDR1, MRP2, MRP3 and BCRP in cryopreserved human hepatocytes in sandwich culture. Xenobiotica 45 : 177-187. 
Bathena SP, Mukherjee S, Olivera M, and Alnouti Y (2013) The profile of bile acids and their sulfate metabolites in human urine and serum. J Chromatogr B Analyt Technol Biomed Life Sci 942-943:53-62.

Bathena SP, Thakare R, Gautam N, Mukherjee S, Olivera M, Meza J, and Alnouti Y (2015) Urinary bile acids as biomarkers for liver diseases II. Signature profiles in patients. Toxicol Sci 143:308-318.

Bednarczyk D and Boiselle C (2016) Organic anion transporting polypeptide (OATP)-mediated transport of coproporphyrins I and III. Xenobiotica 46:457-466.

Benjamini Y and Hochberg Y (1995) Controlling the false discovery rate: a practical and powerful approach to multiple testing. J R Statist Soc B 57:289-300.

Bi YA, Kazolias D, and Duignan DB (2006) Use of cryopreserved human hepatocytes in sandwich culture to measure hepatobiliary transport. Drug Metab Dispos 34:1658-1665.

Chu X, Shih SJ, Shaw R, Hentze H, Chan GH, Owens K, Wang S, Cai X, Newton D, Castro-Perez J, et al. (2015) Evaluation of cynomolgus monkeys for the identification of endogenous biomarkers for hepatic transporter inhibition an, $\mathrm{d}$ as a translatable model to predict pharmacokinetic interactions with statins in humans. Drug Metab Dispos 43:851-863.

Clarke JD, Hardwick RN, Lake AD, Lickteig AJ, Goedken MJ, Klaassen CD, and Cherrington NJ (2014) Synergistic interaction between genetics and disease on pravastatin disposition. J Hepato 61:139-147.

Donazzolo E, Gucciardi A, Mazzier D, Peggion C, Pirillo P, Naturale M, Moretto A, and Giordano G (2017) Improved synthesis of glycine, taurine and sulfate conjugated bile acids as reference compounds and internal standards for ESI-MS/MS urinary profiling of inborn errors of bile acid synthesis. Chem Phys Lipids 204:43-56.

Dong Z, Ekins S, and Polli JE (2015) A substrate pharmacophore for the human sodium taurocholate co-transporting polypeptide. Int J Pharm 478:88-95.

Giacomini KM, Huang SM, Tweedie DJ, Benet LZ, Brouwer KL, Chu X, Dahlin A, Evers R, Fischer V, Hillgren KM, et al.; International Transporter Consortium (2010) Membrane transporters in drug development. Nat Rev Drug Discov 9:215-236.

Gong IY and Kim RB (2013) Impact of genetic variation in OATP transporters to drug disposition and response. Drug Metab Pharmacokinet 28:4-18.

Ho RH, Leake BF, Roberts RL, Lee W, and Kim RB (2004) Ethnicity-dependent polymorphism in $\mathrm{Na}^{+}$-taurocholate cotransporting polypeptide (SLC10A1) reveals a domain critical for bile acid substrate recognition. J Biol Chem 279:7213-7222.

Jamei M, Bajot F, Neuhoff S, Barter Z, Yang J, Rostami-Hodjegan A, and Rowland-Yeo K (2014) A mechanistic framework for in vitro-in vivo extrapolation of liver membrane transporters: prediction of drug-drug interaction between rosuvastatin and cyclosporine. Clin Pharmacokine 53:73-87.

Kobayashi N, Katsumata H, Uto Y, Goto J, Niwa T, Kobayashi K, and Mizuuchi Y (2002) A monoclonal antibody-based enzyme-linked immunosorbent assay of glycolithocholic acid sulfate in human urine for liver function test. Steroids 67:827-833.

Lai Y, Mandlekar S, Shen H, Holenarsipur VK, Langish R, Rajanna P, Murugesan S, Gaud N, Selvam S, Date O, et al. (2016) Coproporphyrins in plasma and urine can be appropriate clinical biomarkers to recapitulate drug-drug interactions mediated by organic anion transporting polypeptide inhibition. J Pharmacol Exp Ther 358:397-404.

Leblanc M, Labrie C, Bélanger A, Candas B, and Labrie F (2003) Bioavailability and pharmacokinetics of dehydroepiandrosterone in the cynomolgus monkey. J Clin Endocrinol Metab $\mathbf{8 8}$ 4293-4302.

Maeda K, Kambara M, Tian Y, Hofmann AF, and Sugiyama Y (2006) Uptake of ursodeoxycholate and its conjugates by human hepatocytes: role of $\mathrm{Na}(+)$-taurocholate cotransporting polypeptide (NTCP), organic anion transporting polypeptide (OATP) 1B1 (OATP-C), and oatp1B3 (OATP8). Mol Pharm 3:70-77.

Marschall HU, Wagner M, Zollner G, Fickert P, Diczfalusy U, Gumhold J, Silbert D, Fuchsbichler A, Benthin L, Grundström R, et al. (2005) Complementary stimulation of hepatobiliary transport and detoxification systems by rifampicin and ursodeoxycholic acid in humans. Gastroenterology 129:476-485.

Meier PJ, Eckhardt U, Schroeder A, Hagenbuch B, and Stieger B (1997) Substrate specificity of sinusoidal bile acid and organic anion uptake systems in rat and human liver. Hepatology $\mathbf{2 6}$ $1667-1677$.

Meng LJ, Wang P, Wolkoff AW, Kim RB, Tirona RG, Hofmann AF, and Pang KS (2002) Transport of the sulfated, amidated bile acid, sulfolithocholyltaurine, into rat hepatocytes is mediated by Oatp1 and Oatp2. Hepatology 35:1031-1040.

Nanashima A, Obatake M, Sumida Y, Abo T, Yamane Y, Nomura M, Yuhio I, Sawai T, Takeshita H, Hidaka S, et al. (2009) Clinical significance of measuring urinary sulfated bile acids in adul patients with hepatobiliary diseases. Hepatogastroenterology 56:299-302.

Nishimura M, Koeda A, Morikawa H, Satoh T, Narimatsu S, and Naito S (2009) Tissue-specific mRNA expression profiles of drug-metabolizing enzymes and transporters in the cynomolgus monkey. Drug Metab Pharmacokinet 24:139-144.

Nishimura M, Koeda A, Shimizu T, Nakayama M, Satoh T, Narimatsu S, and Naito S (2008) Comparison of inducibility of sulfotransferase and UDP-glucuronosyltransferase mRNAs by prototypical microsomal enzyme inducers in primary cultures of human and cynomolgus monkey hepatocytes. Drug Metab Pharmacokinet 23:45-53.
Poirier A, Funk C, Lavé T, and Noé J (2007) New strategies to address drug-drug interactions involving OATPs. Curr Opin Drug Discov Devel 10:74-83.

Rodrigues AD, Lai Y, Cvijic ME, Elkin LL, Zvyaga T, and Soars MG (2014) Drug-induced perturbations of the bile acid pool, cholestasis, and hepatotoxicity: mechanistic considerations beyond the direct inhibition of the bile salt export pump. Drug Metab Dispos 42:566-574.

Sasaki M, Suzuki H, Ito K, Abe T, and Sugiyama Y (2002) Transcellular transport of organic anions across a double-transfected Madin-Darby canine kidney II cell monolayer expressing both human organic anion-transporting polypeptide (OATP2/SLC21A6) and Multidrug resistanceassociated protein 2 (MRP2/ABCC2). J Biol Chem 277:6497-6503.

Shen H, Dai J, Liu T, Cheng Y, Chen W, Freeden C, Zhang Y, Humphreys WG, Marathe P, and Lai Y (2016) Coproporphyrins I and III as functional markers of OATP1B activity: in vitro and in vivo evaluation in preclinical species. J Pharmacol Exp Ther 357:382-393.

Shen H, Su H, Liu T, Yao M, Mintier G, Li L, Fancher RM, Iyer R, Marathe P, Lai Y, et al. (2015) Evaluation of rosuvastatin as an organic anion transporting polypeptide (OATP) probe substrate: in vitro transport and in vivo disposition in cynomolgus monkeys. J Pharmacol Exp Ther 353: 380-391.

Shen H, Yang Z, Mintier G, Han YH, Chen C, Balimane P, Jemal M, Zhao W, Zhang R, Kallipatti $\mathrm{S}$, et al. (2013) Cynomolgus monkey as a potential model to assess drug interactions involving hepatic organic anion transporting polypeptides: in vitro, in vivo, and in vitro-to-in vivo extrapolation. J Pharmacol Exp Ther 344:673-685.

Suga T, Yamaguchi H, Sato T, Maekawa M, Goto J, and Mano N (2017) Preference of conjugated bile acids over unconjugated bile acids as substrates for OATP1B1 and OATP1B3. PLoS One 12:e0169719 DOI: [published ahead of print].

Tahara H, Shono M, Kusuhara H, Kinoshita H, Fuse E, Takadate A, Otagiri M, and Sugiyama Y (2005) Molecular cloning and functional analyses of OAT1 and OAT3 from cynomolgus monkey kidney. Pharm Res 22:647-660.

Takahashi T, Ohtsuka T, Yoshikawa T, Tatekawa I, Uno Y, Utoh M, Yamazaki H, and Kume T (2013) Pitavastatin as an in vivo probe for studying hepatic organic anion transporting polypeptide-mediated drug-drug interactions in cynomolgus monkeys. Drug Metab Dispos 41: $1875-1882$.

Tsuruya Y, Kato K, Sano Y, Imamura Y, Maeda K, Kumagai Y, Sugiyama Y, and Kusuhara H (2016) Investigation of endogenous compounds applicable to drug-drug interaction studies involving the renal organic anion transporters, OAT1 and OAT3, in humans. Drug Metab Dispos 44:1925-1933.

Vaidyanathan J, Yoshida K, Arya V, and Zhang L (2016) Comparing various in vitro prediction criteria to assess the potential of a new molecular entity to inhibit organic anion transporting polypeptide 1B1. J Clin Pharmacol 56 (Suppl 7):S59-S72.

Wang L, Prasad B, Salphati L, Chu X, Gupta A, Hop CE, Evers R, and Unadkat JD (2015) Interspecies variability in expression of hepatobiliary transporters across human, dog, monkey, and rat as determined by quantitative proteomics. Drug Metab Dispos 43:367-374.

Watanabe M, Watanabe T, Yabuki M, and Tamai I (2015) Dehydroepiandrosterone sulfate, a useful endogenous probe for evaluation of drug-drug interaction on hepatic organic anion transporting polypeptide (OATP) in cynomolgus monkeys. Drug Metab Pharmacokinet 30: 198-204.

West BT, Welch KB, and Galecki AT (2015) Models for Repeated-Measures Data: The Rat Brain Example, in Linear-Mixed Models-A Practical Guide Using Statistical Software. West BT, Welch KB, and, Galecki AT, eds. 2nd edition, pp 232, Taylor and Francis/CRC Press, Boca Raton, FL. Yee SW, Giacomini MM, Hsueh CH, Weitz D, Liang X, Goswami S, Kinchen JM, Coelho A, Zur AA, Mertsch K, et al. (2016) Metabolomic and genome-wide association studies reveal potential endogenous biomarkers for OATP1B1. Clin Pharmacol Ther 100:524-536.

Yoshida K, Maeda K, and Sugiyama Y (2012) Transporter-mediated drug-drug interactions involving OATP substrates: predictions based on in vitro inhibition studies. Clin Pharmacol Ther 91:1053-1064.

Yoshikado T, Yoshida K, Kotani N, Nakada T, Asaumi R, Toshimoto K, Maeda K, Kusuhara H, and Sugiyama Y (2016) Quantitative analyses of hepatic OATP-mediated interactions between statins and inhibitors using PBPK modeling with a parameter optimization method. Clin Pharmacol Ther 100:513-523.

Zelcer N, Reid G, Wielinga P, Kuil A, van der Heijden I, Schuetz JD, and Borst P (2003b) Steroid and bile acid conjugates are substrates of human multidrug-resistance protein (MRP) 4 (ATPbinding cassette C4). Biochem J 371:361-367.

Zelcer N, Saeki T, Bot I, Kuil A, and Borst P (2003a) Transport of bile acids in multidrugresistance-protein 3-overexpressing cells co-transfected with the ileal $\mathrm{Na}^{+}$-dependent bile-acid transporter. Biochem J 369:23-30.

Address correspondence to: Dr. A. David Rodrigues, Pharmacokinetics, Dynamics, \& Metabolism, Medicine Design, Eastern Point Road, Bldg 220/002/2565, Mail Stop 8220-2439, Pfizer Inc., Groton, CT 06340. E-mail: a. david.rodrigues@pfizer.com 